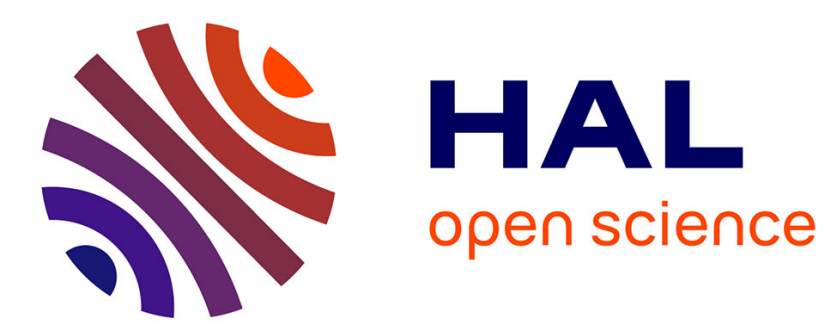

\title{
From averaged to simultaneous controllability
}

Jérôme Lohéac, Enrique Zuazua

\section{To cite this version:}

Jérôme Lohéac, Enrique Zuazua. From averaged to simultaneous controllability. Annales de la Faculté des Sciences de Toulouse. Mathématiques., 2016, 25 (4), pp.785 - 828. 10.5802/afst.1511 . hal01164525v3

\section{HAL Id: hal-01164525 \\ https://hal.science/hal-01164525v3}

Submitted on 6 Dec 2016

HAL is a multi-disciplinary open access archive for the deposit and dissemination of scientific research documents, whether they are published or not. The documents may come from teaching and research institutions in France or abroad, or from public or private research centers.
L'archive ouverte pluridisciplinaire HAL, est destinée au dépôt et à la diffusion de documents scientifiques de niveau recherche, publiés ou non, émanant des établissements d'enseignement et de recherche français ou étrangers, des laboratoires publics ou privés. 


\title{
From averaged to simultaneous controllability*
}

\author{
Jérôme Lohéac ${ }^{\mathrm{a}} \quad$ Enrique Zuazua ${ }^{\mathrm{b}}$ \\ ${ }^{a}$ LUNAM Université, IRCCyN UMR CNRS 6597 (Institut de Recherche en Communications et Cybernétique de \\ Nantes), École des Mines de Nantes, 4 rue Alfred Kastler, 44307 Nantes - France \\ b Departamento de Matemáticas, Universidad Autónoma de Madrid, Cantoblanco, 28049 Madrid - Spain
}

\begin{abstract}
We consider a linear finite dimensional control system depending on unknown parameters. We aim to design controls, independent of the parameters, to control the system in some optimal sense. We discuss the notions of averaged control, according to which one aims to control only the average of the states with respect to the unknown parameters, and the notion of simultaneous control in which the goal is to control the system for all values of these parameters. We show how these notions are connected through a penalization process. Roughly, averaged control is a relaxed version of the simultaneous control property, in which the differences of the states with respect to the various parameters are left free, while simultaneous control can be achieved by reinforcing the averaged control property by penalizing these differences. We show however that these two notions require of different rank conditions on the matrices determining the dynamics and the control. When the stronger conditions for simultaneous control are fulfilled, one can obtain the later as a limit, through this penalization process, out of the averaged control property.
\end{abstract}

\section{Résumé}

Nous considérons un système de contrôle linéaire de dimension finie dépendant de paramètres inconnus. L'objectif est de construire des contrôles indépendants des paramètres afin de contrôler le système en un sens optimal. Nous discutons la notion de contrôle moyenné, dont le but est de contrôler seulement la moyenne des états par rapport aux paramètres, ainsi que la notion de contrôle simultané, dont l'objectif est de contrôler pour chaque paramètre l'état du système associé à ce paramètre. Nous montrerons que ces deux notions peuvent être connectées par le biais d'un processus de pénalisation. Plus précisément, la propriété de contrôlabilité en moyenne est une relaxation de la propriété de contrôlabilité simultanée. Pour la notion de contrôlabilité en moyenne les écarts entre les états par rapport aux paramètres sont laissés libres tandis que ces derniers sont forcés pour la notions de contrôlabilité simultanée. Afin de relier le contrôle moyenné au contrôle simultané, ce seront ces écarts qui seront pénalisés. Cependant, ces deux notions de contrôle requirent différentes conditions sur les rangs des matrices déterminant la dynamique du système et le contrôle. Lorsque la condition de rang pour le contrôle simultané est satisfaite, nous montrerons que le contrôle simultané peut être obtenu à partir du contrôle moyenné, comme limite de ce processus de pénalisation.

${ }^{*}$ This work was supported by the Advanced Grants NUMERIWAVES/FP7-246775 of the European Research Council Executive Agency, FA9550-14-1-0214 of the EOARD-AFOSR, PI2010-04 and the BERC 2014-2017 program of the Basque Government, the MTM2011-29306-C02-00 and SEV-2013-0323 Grants of the MINECO.

The first author thanks BCAM for its hospitality and support as a Visiting fellow of the NUMERIWAVES Advanced Grant of the ERC.

The authors thank the CIMI - Toulouse for the hospitality and support during the preparation of this work in the context of the Excellence Chair in "PDE, Control and Numerics".

Email addresses: Jerome.Loheac@irccyn.ec-nantes.fr (J. Lohéac), enrique.zuazua@uam.es (E. Zuazua). 
AMS subject classification (MSC 2010): 49J55, 34H05, 49J15, 93C05, 93C73, 49N05.

Key words: Controllability, parameter dependent system, averaged control, simultaneous control, penalization.

\section{Introduction}

We consider a parameter dependent control system:

$$
\begin{aligned}
\dot{y}_{\zeta} & =A_{\zeta} y_{\zeta}+B_{\zeta} u \quad(t \in(0, T)), \\
y_{\zeta}(0) & =y_{\zeta}^{i} .
\end{aligned}
$$

In order to fix the notation, all along this paper, $\zeta \in \Omega$ is a random parameter (the system's parameter) following a probability law $\mu$, with $(\Omega, \mathcal{F}, \mu)$ a probability space (in particular, $\mu(\Omega)=1$ ), $X=\mathbb{R}^{n}$ is the state space and $U=\mathbb{R}^{m}$ the control one. We assume that for every $\zeta \in \Omega, A_{\zeta} \in \mathcal{L}(X)$ and $B_{\zeta} \in \mathcal{L}(U, X)$.

The control $t \mapsto u(t) \in U$ is assumed to be independent of the parameter $\zeta$ whereas the state $y_{\zeta}(t)=y_{\zeta}(t ; u) \in X$ is time and parameter dependent. In addition, by Duhamel formula, $y_{\zeta}$ can be represented as follows:

$$
y_{\zeta}(t ; u)=e^{t A_{\zeta}} \mathrm{y}_{\zeta}^{i}+\int_{0}^{T} e^{(t-s) A_{\zeta}} B_{\zeta} u(s) \mathrm{d} s \quad\left(\zeta \in \Omega, t \geqslant 0, u \in L_{l o c}^{2}\left(\mathbb{R}_{+}, U\right)\right) .
$$

Let us also define the space:

$$
L^{2}(\Omega, X ; \mu)=\left\{\left(\mathrm{y}_{\zeta}\right)_{\zeta} \in X^{\Omega}, \int_{\Omega}\left\|\mathrm{y}_{\zeta}\right\|_{X}^{2} \mathrm{~d} \mu_{\zeta}\right\}
$$

which is an Hilbert space endowed with the scalar product:

$$
\left\langle\mathrm{y}_{\zeta}, \mathrm{z}_{\zeta}\right\rangle_{L^{2}(\Omega, X ; \mu)}=\int_{\Omega}\left\langle\mathrm{y}_{\zeta}, \mathrm{z}_{\zeta}\right\rangle_{X} \mathrm{~d} \mu_{\zeta} \quad\left(\left(\mathrm{y}_{\zeta}\right)_{\zeta},\left(\mathrm{z}_{\zeta}\right)_{\zeta} \in L^{2}(\Omega, X ; \mu)\right)
$$

In section 2 we introduce precise conditions on $\zeta \mapsto\left(A_{\zeta}, B_{\zeta}\right)$ ensuring that for every $t \geqslant 0$ and every $u \in L_{l o c}^{2}\left(\mathbb{R}_{+}, U\right),\left(y_{\zeta}(t ; u)\right)_{\zeta} \in L^{2}(\Omega, X ; \mu)$ whenever the parameter-dependent initial data satisfy $\left(\mathrm{y}_{\zeta}^{i}\right)_{\zeta} \in L^{2}(\Omega, X ; \mu)$.

This paper is devoted to analyse the following controllability problems.

- Averaged controllability: The system is said to be averaged controllable in time $T>0$ if, for every $\left(\mathrm{y}_{\zeta}^{i}\right)_{\zeta} \in L^{2}(\Omega, X ; \mu)$ and every $\mathrm{y}^{f} \in X$, there exists $u \in L^{2}([0, T], U)$ such that:

$$
\int_{\Omega} y_{\zeta}(T ; u) \mathrm{d} \mu_{\zeta}=\mathrm{y}^{f}
$$

In other words, averaged controllability is the control of the expectation of the system's output. This notion is illustrated on Figure 1a.

- Exact simultaneous controllability: The system is said to be exactly simultaneously controllable in time $T>0$ if, for every $\left(\mathrm{y}_{\zeta}^{i}\right)_{\zeta},\left(\mathrm{y}_{\zeta}^{f}\right)_{\zeta} \in L^{2}(\Omega, X ; \mu)$, there exists $u \in L^{2}([0, T], U)$ such that:

$$
y_{\zeta}(T ; u)=\mathrm{y}_{\zeta}^{f} \quad(\zeta \in \Omega \quad \mu \text {-a.e. })
$$

This notion is illustrated on Figure $1 b$ 
- Approximate simultaneous controllability: The system is said to be approximately simultaneously controllable in time $T>0$ if, for every $\left(\mathrm{y}_{\zeta}^{i}\right)_{\zeta},\left(\mathrm{y}_{\zeta}^{f}\right)_{\zeta} \in L^{2}(\Omega, X ; \mu)$ and every $\varepsilon>0$, there exists $u \in L^{2}([0, T], U)$ such that:

$$
\int_{\Omega}\left\|y_{\zeta}(T ; u)-\mathrm{y}_{\zeta}^{f}\right\|_{X}^{2} \mathrm{~d} \mu_{\zeta} \leqslant \varepsilon
$$

This notion is illustrated on Figure 1c.

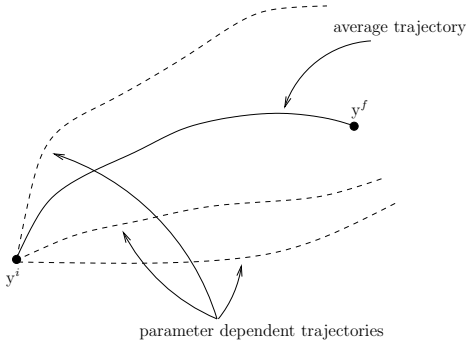

(a) Averaged controllability.

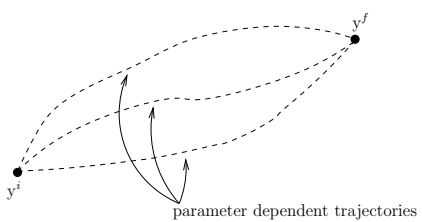

(b) Simultaneous controllability.

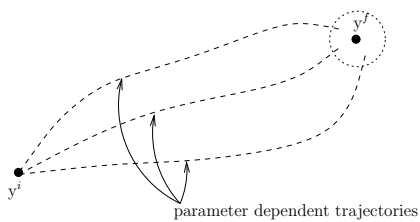

(c) Approximate Simultaneous controllability.

Figure 1: Different controllability notions, introduced in $(1.5),(1.6)$ and $(1.7)$, for parameter dependent systems, with initial condition and target independent of $\zeta$.

Remark 1.1. 1. Even if the system (1.1) is controllable in average, this fact does not give any information on the variance of the outputs.

2. There is no natural ordinary differential equation describing the average $Y(t)=\int_{\Omega} y_{\zeta}(t) \mathrm{d} \mu_{\zeta}$, except when $A_{\zeta}$ is independent of $\zeta$ for which we have: $\dot{Y}=A Y+\left(\int_{\Omega} B_{\zeta} \mathrm{d} \mu_{\zeta}\right) u$. In this particular case, the averaged controllability property is equivalent to the controllability of the pair $\left(A, \int_{\Omega} B_{\zeta} \mathrm{d} \mu_{\zeta}\right)$.

3. It is obvious that the exact simultaneous controllability property implies the averaged controllability and the approximate simultaneous controllability ones. In addition, one can find systems which are controllable in average (resp. approximatively simultaneously controllable) which are not exactly simultaneously controllable, see for instance Example 4.2 (resp. Example 3.1).

Moreover, the approximate simultaneous controllability property implies the averaged controllability one. In fact, the approximate simultaneous controllability property ensures that given $T>0,\left(\mathrm{y}_{\zeta}^{i}\right)_{\zeta} \in$ $L^{2}(\Omega, X ; \mu), \mathrm{y}^{f} \in X$ and $\varepsilon>0$, there exists $u^{\varepsilon} \in L^{2}([0, T], U)$ such that

$$
\left\|y_{\zeta}\left(T ; u^{\varepsilon}\right)-\mathrm{y}^{f}\right\|_{L^{2}(\Omega, X ; \mu)}^{2} \leqslant \varepsilon .
$$

But, by Cauchy-Schwarz inequality,

$$
\left\|\int_{\Omega}\left(y_{\zeta}\left(T ; u^{\varepsilon}\right)-\mathrm{y}^{f}\right) \mathrm{d} \mu_{\zeta}\right\|_{X}^{2} \leqslant \int_{\Omega}\left\|y_{\zeta}\left(T ; u^{\varepsilon}\right)-\mathrm{y}^{f}\right\|_{X}^{2} \mathrm{~d} \mu_{\zeta} .
$$


Thus, the system is approximatively controllable in average i.e. the linear and continuous map $\Phi: u \in L^{2}([0, T], U) \mapsto \int_{\Omega} \int_{0}^{T} e^{(t-t) A_{\zeta}} B_{\zeta} u(t) \mathrm{d} t \mathrm{~d} \mu_{\zeta} \in X$ has a dense image in $X$. But since $X$ is a finite dimensional vector space, we obtain $\operatorname{Im} \Phi=X$, i.e. the system is controllable in average.

A proof using probabilistic arguments can also be given. More precisely, approximate simultaneous controllability in time $T>0$ means that for every $\left(\mathrm{y}_{\zeta}^{f}\right)_{\zeta} \in L^{2}(\Omega, X ; \mu)$ and every $\varepsilon>0$, there exists $u^{\varepsilon} \in L^{2}([0, T], U)$ such that $\left\|y_{\zeta}\left(T ; u^{\varepsilon}\right)-\mathrm{y}_{\zeta}^{f}\right\|_{L^{2}(\Omega, X ; \mu)} \leqslant \varepsilon$, that is to say that the sequence of random variables $\left(\left(y_{\zeta}\left(T ; u^{\varepsilon}\right)\right)_{\zeta}\right)_{\varepsilon}$ converges in mean square to the random variable $\left(\mathrm{y}_{\zeta}^{f}\right)_{\zeta}$ as $\varepsilon$ goes to 0 . This convergence in mean square implies the convergence in law and hence the convergence of all finite momenta and, in particular,

$$
\lim _{\varepsilon \rightarrow 0} \int_{\Omega} y_{\zeta}\left(T ; u^{\varepsilon}\right) \mathrm{d} \mu_{\zeta}=\int_{\Omega} \mathrm{y}_{\zeta}^{f} \mathrm{~d} \mu_{\zeta}
$$

In the spirit of the above paragraph, if there exists $u \in L^{2}([0, T], U)$ such that the family of random variables $\left(\left(y_{\zeta}(t ; u)\right)_{\zeta}\right)_{t \in[0, T]}$ converges in law to $\left(\mathrm{y}_{\zeta}^{f}\right)_{\zeta}$ as $t$ goes to $T$, then the averaged controllability property holds. Let us mention that this convergence in law is a weaker notion than the exact simultaneous controllability one (corresponding to the mean square convergence).

4. When $\Omega=\left\{\zeta_{1}, \cdots, \zeta_{K}\right\}$ is of finite cardinal, the simultaneous controllability is equivalent to the classical controllability one for the augmented system:

$$
\dot{\boldsymbol{y}}=\mathbf{A} \boldsymbol{y}+\mathbf{B} u
$$

with:

$$
\boldsymbol{y}=\left(\begin{array}{c}
y_{\zeta_{1}} \\
\vdots \\
y_{\zeta_{K}}
\end{array}\right), \quad \mathbf{A}=\left(\begin{array}{ccc}
A_{\zeta_{1}} & & 0 \\
& \ddots & \\
0 & & A_{\zeta_{K}}
\end{array}\right) \quad \text { and } \quad \mathbf{B}=\left(\begin{array}{c}
B_{\zeta_{1}} \\
\vdots \\
B_{\zeta_{K}}
\end{array}\right) \text {. }
$$

And the controllability of this system is equivalent to the Kalman rank condition:

$$
\operatorname{rank}\left[\begin{array}{llll}
\mathbf{B} & \mathbf{A B} & \cdots & \mathbf{A}^{K \operatorname{dim} X-1} \mathbf{B}
\end{array}\right]=K \operatorname{dim} X .
$$

5. In the previous item, we have seen that the simultaneous controllability property when the cardinal of $\Omega$ is finite can be interpreted in terms of a classical rank condition. But, when $\Omega$ is infinite, the output of the system is the function $\zeta \in \Omega \mapsto y_{\zeta}(T) \in X$, living in an infinite-dimensional space. The first issue to be addressed is the choice of the norm in that space.

In the following, we choose the $L^{2}$-norm. Accordingly, the fact that $y_{\zeta}(T)=\mathrm{y}_{\zeta}^{f}$ holds for almost every $\zeta \in \Omega$ with respect to the measure $\mu$ is guaranteed by the fact that $\int_{\Omega}\left\|y_{\zeta}(T)-\mathrm{y}_{\zeta}^{f}\right\|_{X}^{2} \mathrm{~d} \mu_{\zeta}=0$. This choice is natural, since in the particular case where $\mathrm{y}_{\zeta}^{f}=\mathrm{y}^{f}$ is independent of $\zeta$ and $\int_{\Omega} y_{\zeta}(T) \mathrm{d} \mu_{\zeta}=\mathrm{y}^{f}$, the integral $\int_{\Omega}\left\|y_{\zeta}(T)-\mathrm{y}_{\zeta}^{f}\right\|_{X}^{2} \mathrm{~d} \mu_{\zeta}$ is the variance of the system's output.

Thus, the $L^{2}$-norm approach is natural from a probabilistic point of view but one could also use any $L^{p}(\Omega, X ; \mu)$-norm. In the next item, we mention some existing literature when considering the $L^{\infty}$-norm. 
6. For parameter dependent systems, the notion of ensemble controllability is also commonly used (see for instance [5, 6, 13, 17]). A system is said to be ensemble controllable in time $T>0$ if, for every $\varepsilon$ and every $\mathrm{y}_{\zeta}^{i}, \mathrm{y}_{\zeta}^{f} \in X$, there exists $u \in L^{2}([0, T], U)$ such that:

$$
\left\|y_{\zeta}(T ; u)-\mathrm{y}_{\zeta}^{f}\right\|_{X} \leqslant \varepsilon \quad(\zeta \in \Omega) \text {. }
$$

This notion of ensemble controllability, which does not seem to have a probabilistic interpretation, is similar to our notion of approximate simultaneous controllability above, where the $L^{2}(\Omega, X ; \mu)$-norm is replaced by the $L^{\infty}(\Omega, X)$ one.

In [3], U. Helmke and M. Schönlein extended this notion to the one of $L^{p}$-ensemble controllability, for $p \in[1, \infty]$. For $p=2$, the $L^{2}$-ensemble controllability corresponds to our approximate simultaneous controllability defined by (1.7). More precisely, the system (1.1) is said $L^{p}$-ensemble controllable if for every $\varepsilon>0$, there exists $u:[0, T] \rightarrow \mathbb{R}$ such that:

$$
\left\|\zeta \mapsto\left(y_{\zeta}(T ; u)-\mathrm{y}_{\zeta}^{f}\right)\right\|_{L^{p}(\Omega, X)} \leqslant \varepsilon
$$

In [3], with $\Omega=\left[\zeta^{-}, \zeta^{+}\right]$a compact subset of $\mathbb{R}$ and $\mu$ the Lebesgue measure, the authors give a necessary and sufficient conditions for the system (1.1) to be $L^{p}$-ensemble controllable.

Controlling the average (or the expectation) of a parameter dependent system is not a new problem. It has been previously studied when a classical control system is perturbed by an additional drift (V. A. Ugrinovskii [18, A. V. Savkin and I. R. Petersen [12, I. R. Petersen [10]). We present here a different frame for which the uncertainty is inside the system itself, and not due to some external noise. Taking into account that we only know the probability distribution of the unknown parameter, it is natural to try to control the expectation of the output of the system.

In [19], it has been shown that the averaged controllability property is equivalent to a Kalman rank condition of infinite order. However, even if the average of the system is controlled, this fact does not ensure that the output of system is close to the desired target for any specific realisation of the parameter. Of course, the ideal situation arises when all the parameter dependent trajectories exactly reach the desired target. This corresponds, precisely, to the notion of simultaneous controllability.

Classically, the simultaneous exact controllability property corresponds, by duality, to the one of simultaneous exact observability (see $\S 3.2$. However, when $\Omega$ is an infinite dimensional set, those properties are difficult to check in practice. This is why, in this article, we show that, if the simultaneous controllability property holds, then the approximate simultaneous control can be achieved from the averaged controls by means of a penalisation procedure and at the limit, when the penalizing parameter goes to $\infty$, we recover the simultaneous control.

The notion of simultaneous controllability was introduced by D. L. Russell [1] (see also J.-L. Lions [7, Chapter 5]) for partial differential equations. As mentioned above, when dealing with finite dimensional systems and when the parameter ranges over a finite set, the problem can be handled through classical rank conditions. However, the issue is much more complex when the parameter ranges over an infinite set.

The averaged controllability property has already been tackled by E. Zuazua et al [19, 4, 9] for some relevant PDE models. However, the link between the averaged and simultaneous controllability in that setting has not been yet developed. The tools developed here could be used to handle PDE and, in general, infinite-dimensional systems, but this requires further efforts. 
In general, the simultaneous controllability problem is set in an infinite dimensional space (this holds when the cardinal of $\Omega$ is infinite). In infinite dimensional spaces the choice of the norm is important and an appropriate choice has to be done. According to the $5^{\text {h }}$ item of Remark 1.1, we chose the weighted $L^{2}$-norm, that corresponds to the variance. More precisely, the simultaneous controllability property (1.6) holds if:

$$
\int_{\Omega}\left\|y_{\zeta}(T)-\mathrm{y}_{\zeta}^{f}\right\|_{X}^{2} \mathrm{~d} \mu_{\zeta}=0
$$

Consequently, in section 4, we introduce the parametrized optimal control problems:

$$
\begin{array}{cl}
\min & \mathcal{J}_{\kappa}(u)=\frac{1}{2} \int_{0}^{T}\|u(t)\|_{U}^{2} \mathrm{~d} t+\kappa \int_{\Omega}\left\|y_{\zeta}(T ; u)-\mathrm{y}_{\zeta}^{f}\right\|_{X}^{2} \mathrm{~d} \mu_{\zeta} \\
& \int_{\Omega} y_{\zeta}(T ; u) \mathrm{d} \mu_{\zeta}=\int_{\Omega} \mathrm{y}_{\zeta}^{f} \mathrm{~d} \mu_{\zeta}
\end{array} \quad(\kappa \geqslant 0),
$$

with $y_{\zeta}$ the solution of 1.1 with control $u$ and initial condition $\mathrm{y}_{\zeta}^{i}$.

We will see in Theorem 4.1 that, at the limit $\kappa \rightarrow \infty$, the minimum $u_{\kappa}$ is a control which minimizes the variance of the system's outputs. For instance, we will see that if the sequence $\left(\mathcal{J}_{\kappa}\left(u_{\kappa}\right)\right)_{\kappa}$ is bounded then the sequence $\left(u_{\kappa}\right)_{\kappa}$ converges to a control $u_{\infty}$ which solves the minimisation problem:

$$
\begin{array}{ll}
\min & \frac{1}{2} \int_{0}^{T}\|u(t)\|_{U}^{2} \mathrm{~d} t \\
& \int_{\Omega}\left\|y_{\zeta}(T ; u)-\mathrm{y}_{\zeta}^{f}\right\|_{X}^{2} \mathrm{~d} \mu_{\zeta}=0 .
\end{array}
$$

\begin{tabular}{|c|c|c|c|}
\hline & \multicolumn{2}{|c|}{$\left(\left\|y_{\zeta}\left(T ; u_{\kappa}\right)-\mathrm{y}_{\zeta}^{f}\right\|_{L^{2}(\Omega, X ; \mu)}\right)_{\kappa}$} \\
\hline & & converge to 0 & do not converge to 0 \\
\hline \multirow{2}{*}{ 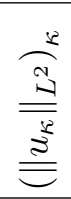 } & bounded & simultaneous exact controllability & $\begin{array}{l}\text { simultaneous exact controllability } \\
\text { to } y_{\zeta}^{\star}\end{array}$ \\
\hline & unbounded & $\begin{array}{l}\text { simultaneous approximate controlla- } \\
\text { bility }\end{array}$ & $\begin{array}{l}\text { simultaneous approximate controlla- } \\
\text { bility to } y_{\zeta}^{\star}\end{array}$ \\
\hline
\end{tabular}

In other words, $u_{\infty}$ is the HUM control (the control obtained from the Hilbert Uniqueness Method) for the simultaneous control problem.

More generally, the result of Theorem 4.1 can be summarized in Table 1, where we have defined $\left(\mathrm{y}_{\zeta}^{\star}\right)_{\zeta} \in L^{2}(\Omega, X ; \mu)$ as the minimizer of $\left\|\mathrm{y}_{\zeta}-\mathrm{y}_{\zeta}^{f}\right\|_{L^{2}(\Omega, X ; \mu)}$ under the constraints $\int_{\Omega} \mathrm{y}_{\zeta} \mathrm{d} \mu_{\zeta}=\int_{\Omega} \mathrm{y}_{\zeta}^{f} \mathrm{~d} \mu_{\zeta}$ and $\left(\mathrm{y}_{\zeta}\right)_{\zeta} \in \overline{\left\{y_{\zeta}(T ; u), u \in L^{2}([0, T], U)\right\}}$.

Table 1: Possible behaviors as $\kappa \rightarrow \infty$, with $\mathrm{y}_{\zeta}^{\star}$ defined by 4.4.

This penalty argument is natural and has already been used in control theory. In J.-L. Lions [8] it was used to achieve approximate controllability as the limit of a sequence of optimal control problems (see also L. A. Fernández and E. Zuazua [2] for semi-linear heat equations). This penalty method has also been used numerically, for the numerical approximation of null controls for parabolic problems (see F. Boyer [1]).

This paper is organized as follows. 
In section 2, we give some conditions on $A_{\zeta}, B_{\zeta}$ and $\mathrm{y}_{\zeta}^{i}$ ensuring that the problem we are considering is well defined. Then, in section 3, we recall some known results about averaged controllability and we describe the duality approach for simultaneous controllability. In section 4, we present the penalty method and give some convergence results. More precisely, in this section we prove the main theorem (Theorem 4.1) of this article. Then, in section 5, we present some results for a further numerical development of the case where $\Omega$ is a countable set. Finally, in section 6 , we conclude this work by some general remarks and open problems.

\section{Admissibility conditions}

In this section, we give some conditions ensuring that $\int_{\Omega} y_{\zeta}(t) \mathrm{d} \mu_{\zeta}$ and $\int_{\Omega}\left\|y_{\zeta}(t)\right\|^{2} \mathrm{~d} \mu_{\zeta}$ are well defined.

Let us consider the Hilbert space $L^{2}(\Omega, X ; \mu)$ defined by $(1.3)$. Using Cauchy-Schwarz together with $\int_{\Omega} \mathrm{d} \mu_{\zeta}=1$, leads to:

$$
\left\|\int_{\Omega} \mathrm{y}_{\zeta} \mathrm{d} \mu_{\zeta}\right\|_{X}^{2} \leqslant\left\|\mathrm{y}_{\zeta}\right\|_{L^{2}(\Omega, X ; \mu)}^{2} \quad\left(\left(\mathrm{y}_{\zeta}\right)_{\zeta} \in L^{2}(\Omega, X ; \mu)\right) .
$$

Thus, in this paragraph, we only give conditions on $A_{\zeta}, B_{\zeta}$ and $\mu$ such that $\left\|y_{\zeta}(t)\right\|_{L^{2}(\Omega, X ; \mu)}<\infty$ and in all this article, we assume that initial and final condition are elements of $L^{2}(\Omega, X ; \mu)$.

By Duhamel formula, the solution $y_{\zeta}(t)=y_{\zeta}(t ; u)$ of $(1.1)$ is given by $(1.2)$, i.e.,

$$
y_{\zeta}(t ; u)=e^{t A_{\zeta}} y_{\zeta}^{i}+\int_{0}^{t} e^{(t-s) A_{\zeta}} B_{\zeta} u(s) \mathrm{d} s \quad(\zeta \in \Omega, t \geqslant 0) .
$$

Lemma 2.1. Set $\left(A_{\zeta}\right)_{\zeta \in \Omega} \in \mathcal{L}(X)^{\Omega}$. For every $T>0$ and every $\zeta \in \Omega$, there exists $\varsigma_{\zeta}(T)>0$ such that:

$$
\left\|e^{T A_{\zeta}^{*}} e^{T A_{\zeta}}\right\|_{X} \leqslant \varsigma_{\zeta}(T)\|\mathrm{y}\|_{X} \quad(\mathrm{y} \in X) .
$$

Assume:

$$
\varsigma_{\zeta}(T)<\infty \quad(\zeta \in \Omega \quad \mu-\text { a.e. }) .
$$

Then for every $T>0$, there exists $\varsigma(T)>0\left(\varsigma(T)=\sup _{\zeta \in \Omega} \varsigma_{\zeta}(T)\right)$ such that:

$$
\left\|e^{T A_{\zeta}} y_{\zeta}^{i}\right\|_{L^{2}(\Omega, X ; \mu)} \leqslant \varsigma(T)\left\|\mathrm{y}_{\zeta}^{i}\right\|_{L^{2}(\Omega, X ; \mu)} \quad\left(\left(\mathrm{y}_{\zeta}^{i}\right)_{\zeta} \in L^{2}(\Omega, X ; \mu)\right)
$$

Proof. The existence of $\varsigma_{\zeta}(T)$ is clear. The result follows from Cauchy-Schwarz inequality.

Example 2.1. If for every $\zeta \in \Omega, A_{\zeta}$ is skew-adjoint, then (2.2) holds with $\varsigma(T)=1$. (In this case, we have $\varsigma_{\zeta}(T)=1$ for every $\zeta \in \Omega$.)

Lemma 2.2. Set $\left(A_{\zeta}\right)_{\zeta \in \Omega} \in \mathcal{L}(X)^{\Omega}$ and $\left(B_{\zeta}\right)_{\zeta \in \Omega} \in \mathcal{L}(U, X)^{\Omega}$. For every $T>0$ and every $\zeta \in \Omega$, there exists a constant $C_{\zeta}(T)>0$ such that:

$$
\left\|\int_{0}^{T} e^{(T-t) A_{\zeta}} B_{\zeta} u(t) \mathrm{d} t\right\|_{X}^{2} \leqslant C_{\zeta}(T)\|u\|_{L^{2}([0, T], U)}^{2} .
$$

Assume that:

$$
\int_{\Omega} C_{\zeta}(T) \mathrm{d} \mu_{\zeta}<\infty
$$


Then for every $T>0$, there exists $C(T)>0$ such that:

$$
\int_{\Omega}\left\|\int_{0}^{T} e^{(T-t) A_{\zeta}} B_{\zeta} u(t) \mathrm{d} t\right\|_{X}^{2} \mathrm{~d} \mu_{\zeta} \leqslant C(T)\|u\|_{L^{2}([0, T], U)}^{2} \quad\left(u \in L^{2}([0, T], U)\right) .
$$

Proof. The existence of $C_{\zeta}(T)>0$ independent of $u$ is classical. The result follows from Minkowski and Cauchy-Schwarz inequalities.

Thus, if $A_{\zeta}$ and $B_{\zeta}$ satisfies the assumption of lemmas 2.1 and 2.2 , then for every $\left(\mathrm{y}_{\zeta}^{i}\right)_{\zeta} \in L^{2}(\Omega, X ; \mu)$, $y(T ; u)$ defined by 1.2 is an element of $L^{2}(\Omega, X ; \mu)$.

From these two lemmas, we can derive the following corollaries:

Corollary 2.1. Assume $\operatorname{Card} \Omega<\infty$ and set $\zeta \in \Omega \mapsto\left(A_{\zeta}, B_{\zeta}\right) \in \mathcal{L}(X) \times \mathcal{L}(U, X)$, then for every $\left(\mathrm{y}_{\zeta}^{i}\right)_{\zeta} \in L^{2}(\Omega, X ; \mu)$, and every $u \in L_{\text {loc }}^{2}\left(\mathbb{R}_{+}, U\right)$, the solution $y_{\zeta}(t ; u)$ of 1.1 belongs to $L^{2}(\Omega, X ; \mu)$ for every $t \geqslant 0$.

Corollary 2.2. Assume $\Omega \subset \mathbb{R}^{d}$ is a bounded set and assume the map $\zeta \mapsto\left(A_{\zeta}, B_{\zeta}\right)$ is continuous on $\overline{\operatorname{co}(\Omega)}$, with $\operatorname{co}(\Omega)$ the smallest convex set containing $\Omega$.

Then for every $\left(\mathrm{y}_{\zeta}^{i}\right)_{\zeta} \in L^{2}(\Omega, X ; \mu)$, every $u \in L_{\text {loc }}^{2}\left(\mathbb{R}_{+}, U\right)$ and every $t \geqslant 0$, the solution $y_{\zeta}(t ; u)$ of (1.1) belongs to $L^{2}(\Omega, X ; \mu)$.

Proof. Since $X$ and $U$ are finite dimensional spaces, for every $\zeta \in \overline{\operatorname{co}(\Omega)}$,

$$
\varsigma_{\zeta}(T):=\sup _{\substack{\mathrm{y} \in X \\\|\mathrm{y}\|_{X}=1}}\left\|e^{T A_{\zeta}^{*}} e^{T A_{\zeta}}\right\|_{X} \quad \text { and } \quad C_{\zeta}(T):=\sup _{\substack{u \in L^{2}([0, T], U) \\\|u\|_{L^{2}([0, T], U)}=1}}\left\|\int_{0}^{T} e^{(T-t) A_{\zeta}} B_{\zeta} u(t) \mathrm{d} t\right\|_{X}^{2}
$$

are well defined for every $\zeta \in \overline{\operatorname{co}(\Omega)}$ and every $T \geqslant 0$.

Moreover, since $\zeta \in \overline{\operatorname{co}(\Omega)} \mapsto\left(A_{\zeta}, B_{\zeta}\right) \in \mathcal{L}(X) \times \mathcal{L}(U, X)$ is continuous, the map $\zeta \in \overline{\operatorname{co}(\Omega)} \mapsto$ $\left(\varsigma_{\zeta}(T), C_{\zeta}(T)\right) \in \mathbb{R}^{2}$ is continuous, thus bounded.

The result follows from lemmas 2.1 and 2.2 .

Remark 2.1. Even if Corollary 2.1 can be proved directly, it can also be seen as a consequence of Corollary 2.2.

Corollary 2.3. Assume $A_{\zeta}$ skew-adjoint for every $\zeta \in \Omega$.

If $\int_{\Omega}\left\|B_{\zeta}\right\|_{\mathcal{L}(U, X)}^{2} \mathrm{~d} \mu_{\zeta}<\infty$, then for every $\left(\mathrm{y}_{\zeta}^{i}\right)_{\zeta} \in L^{2}(\Omega, X ; \mu)$ and every $u \in L_{\text {loc }}^{2}\left(\mathbb{R}_{+}, U\right)$, the solution $y_{\zeta}(t ; u)$ of 1.1 belongs to $L^{2}(\Omega, X ; \mu)$ for every $t \geqslant 0$.

Proof. According to Lemma 2.1 and Example 2.1. we have $\left(e^{t A_{\zeta}} \mathrm{y}_{\zeta}^{i}\right)_{\zeta} \in L^{2}(\Omega, X ; \mu)$. In addition, we have:

$$
\left\|\int_{0}^{t} e^{(t-s) A_{\zeta}} B_{\zeta} u(s) \mathrm{d} s\right\|_{X} \leqslant \int_{0}^{t}\left\|e^{(t-s) A_{\zeta}} B_{\zeta} u(s)\right\|_{X} \mathrm{~d} s=\int_{0}^{t}\left\|B_{\zeta} u(s)\right\|_{X} \mathrm{~d} s \leqslant \sqrt{t}\left\|B_{\zeta}\right\|_{\mathcal{L}(U, X)}\|u\|_{L^{2}([0, t], U)} .
$$

Thus the assumptions of Lemma 2.2 are fulfilled. 


\section{Duality approach and Kalman rank conditions}

Here and in the sequel we assume that the hypotheses of lemmas 2.1 and 2.2 are satisfied.

\subsection{State of the art for averaged controllability}

Let us recall some known results on averaged controllability for finite dimensional systems. These results are taken from [19].

Theorem 3.1 ([19] Theorem 1). System (1.1) fulfills the averaged controllability property (1.5) if and only if the following rank condition is satisfied:

$$
\operatorname{rank}\left[\int_{\Omega}\left(A_{\zeta}\right)^{j} B_{\zeta} \mathrm{d} \mu_{\zeta}, j \geqslant 0\right]=\operatorname{dim} X
$$

This result is based on duality arguments. More precisely, we introduce the (parameter dependent) adjoint system:

$$
\begin{aligned}
-\dot{z}_{\zeta} & =A_{\zeta}^{*} z_{\zeta} \quad(t \in(0, T)), \\
z_{\zeta}(T) & =\mathrm{z}^{f}
\end{aligned}
$$

Notice that even if this system depends of the parameter $\zeta$ the final condition $\mathrm{z}^{f}$ is independent of $\zeta$.

The next result makes the link between averaged controllability, and averaged observability and gives also a link between the adjoint system and the control of minimal $L^{2}$-norm.

Theorem 3.2 ([19] Theorem 2). System (1.1) fulfills the averaged controllability property (1.5) if and only if the adjoint system (3.2) satisfies the averaged observability inequality:

$$
\bar{c}(T)\left\|\mathrm{z}^{f}\right\|_{X}^{2} \leqslant \int_{0}^{T}\left\|\int_{\Omega} B_{\zeta}^{*} z_{\zeta}(t) \mathrm{d} \mu_{\zeta}\right\|_{U}^{2} \mathrm{~d} t \quad\left(\mathrm{z}^{f} \in X\right),
$$

where $\bar{c}(T)>0$ is a constant independent of $\mathrm{z}^{f}$.

In addition, both conditions are equivalent to the rank condition (3.1).

When these properties hold, the averaged control of minimal $L^{2}([0, T], U)$-norm is given by:

$$
u(t)=\int_{\Omega} B_{\zeta}^{*} \bar{z}_{\zeta}(t) \mathrm{d} \mu_{\zeta} \quad(t \in(0, T))
$$

where $\left\{\bar{z}_{\zeta}\right\}_{\zeta}$ is the solution of the adjoint system (3.2) corresponding to the datum $\mathrm{z}^{f} \in X$ minimizing the functional:

$$
\begin{aligned}
J: X & \longrightarrow \mathbb{R} \\
\mathrm{z}^{f} & \longmapsto \frac{1}{2} \int_{0}^{T}\left\|\int_{\Omega} B_{\zeta}^{*} z_{\zeta}(t) \mathrm{d} \mu_{\zeta}\right\|_{U}^{2} \mathrm{~d} t-\left\langle\mathrm{y}^{f}, \mathrm{z}^{f}\right\rangle_{X}+\int_{\Omega}\left\langle\mathrm{y}_{\zeta}^{i}, z_{\zeta}(0)\right\rangle_{X} \mathrm{~d} \mu_{\zeta} .
\end{aligned}
$$




\subsection{Observability inequality for exact simultaneous controllability}

Let us define for every $\zeta \in \Omega$ the adjoint system of 1.1 :

$$
\begin{aligned}
-\dot{z}_{\zeta} & =A_{\zeta}^{*} z_{\zeta} \quad(t \in(0, T)), \\
z_{\zeta}(T) & =\mathrm{z}_{\zeta}^{f}
\end{aligned}
$$

If the system (1.1) is simultaneously controllable then for every $\left(\mathrm{z}_{\zeta}^{f}\right)_{\zeta} \in L^{2}(\Omega, X ; \mu)$,

$$
\left\langle\left(y_{\zeta}(T)-\mathrm{y}_{\zeta}^{f}\right)_{\zeta},\left(\mathrm{z}_{\zeta}^{f}\right)_{\zeta}\right\rangle_{L^{2}(\Omega, X ; \mu)}=0
$$

That is to say:

$$
\int_{0}^{T}\left\langle u(t), \int_{\Omega} B_{\zeta}^{*} z_{\zeta}(t) \mathrm{d} \mu_{\zeta}\right\rangle_{U} \mathrm{~d} t=\left\langle\left(\mathrm{y}_{\zeta}^{f}\right)_{\zeta},\left(\mathrm{z}_{\zeta}^{f}\right)_{\zeta}\right\rangle_{L^{2}(\Omega, X ; \mu)}-\left\langle\left(\mathrm{y}_{\zeta}^{i}\right)_{\zeta},\left(z_{\zeta}(0)\right)_{\zeta}\right\rangle_{L^{2}(\Omega, X ; \mu)} .
$$

Let us then define the cost function $\mathfrak{J}$ by:

$$
\begin{aligned}
\mathfrak{J}: L^{2}(\Omega, X ; \mu) & \longrightarrow \mathbb{R} \\
\left(\mathrm{z}_{\zeta}^{f}\right)_{\zeta} & \longmapsto \frac{1}{2} \int_{0}^{T}\left\|\int_{\Omega} B_{\zeta}^{*} z_{\zeta}(t) \mathrm{d} \mu_{\zeta}\right\|_{U}^{2} \mathrm{~d} t-\int_{\Omega}\left\langle\mathrm{y}_{\zeta}^{f}, \mathrm{z}_{\zeta}^{f}\right\rangle_{X} \mathrm{~d} \mu_{\zeta}+\int_{\Omega}\left\langle\mathrm{y}_{\zeta}^{i}, z_{\zeta}(0)\right\rangle_{X} \mathrm{~d} \mu_{\zeta},
\end{aligned}
$$

where $z_{\zeta}$ is the solution of 3.6 .

The only difference between the cost functions defined by (3.5) for averaged controllability and (3.7) for simultaneous controllability is that, for simultaneous controllability, we allowed the final condition of the adjoint system to depend on the parameter $\zeta$.

Assuming that $\mathfrak{J}$ has a minimizer $\left(\hat{\mathbf{z}}_{\zeta}\right)_{\zeta} \in L^{2}(\Omega, X ; \mu)$, we obtain, by computing the first variation of $\mathfrak{J}$,

$$
\hat{u}(t)=\int_{\Omega} B_{\zeta}^{*} \hat{z}_{\zeta}(t) \mathrm{d} \mu_{\zeta} \quad(t \in[0, T] \quad \text { a.e. }) .
$$

It is clear that $\mathfrak{J}$ is convex. Thus, proving the existence of a minimizer $\left(z_{\zeta}^{f}\right)_{\zeta} \in L^{2}(\Omega, X ; \mu)$ for $\mathfrak{J}$ is equivalent to showing that $\mathfrak{J}$ is coercive, i.e. to the existence of a constant $\hat{c}(T)>0$ such that:

$$
\hat{c}(T) \int_{\Omega}\left\|\mathrm{z}_{\zeta}^{f}\right\|_{X}^{2} \mathrm{~d} \mu_{\zeta} \leqslant \int_{0}^{T}\left\|\int_{\Omega} B_{\zeta}^{*} z_{\zeta}(t) \mathrm{d} \mu_{\zeta}\right\|_{U}^{2} \mathrm{~d} t \quad\left(\left(\mathrm{z}_{\zeta}^{f}\right)_{\zeta} \in L^{2}(\Omega, X ; \mu)\right) .
$$

where $z_{\zeta}$ is the solution of (3.6) with final condition $\mathrm{z}_{\zeta}^{f}$.

Summarizing this discussion, we end up with:

Theorem 3.3. System (1.1) fulfills the exact simultaneous controllability property (1.10) if and only if the adjoint system (3.6) satisfies the exact simultaneous observability inequality (3.9).

When these properties hold, the exact simultaneous control of minimal norm is given by (3.8), where $\hat{z}_{\zeta}$ is the solution of 3.6 with final condition $\hat{\mathbf{z}}_{\zeta}^{f}$ and $\left(\hat{\mathrm{z}}_{\zeta}^{f}\right)_{\zeta} \in L^{2}(\Omega, X ; \mu)$ is the minimizer of $\mathfrak{J}$ defined by 3.7 .

Let us notice that very few systems have the property of simultaneous controllability. When card $\Omega$ is finite, the situation is clear since simultaneous controllability follows from a Kalman rank on an augmented system (see $4^{\mathrm{h}}$ item of Remark 1.1. But when $\operatorname{card} \Omega$ is infinite, the situation more complex and we have from [16, Theorem 3.3.1], 
Proposition 3.1. If $L^{2}(\Omega, X ; \mu)$ is an infinite dimensional space, then the system (1.1) will never be exactly simultaneously controllable.

Example 3.1. Let $\left(\mu_{n}\right)_{n \in \mathbb{N}^{*}}$ be a nonnegative sequence of real numbers and $\left(\zeta_{n}\right)_{n \in \mathbb{N}^{*}}$ of real numbers and assume that $\sum_{n \in \mathbb{N}^{*}} \mu_{n}=1$ and $\zeta_{n} \neq \zeta_{m}$ for every $n \neq m$. Let us then define $\Omega=\left\{\zeta_{n}, n \in \mathbb{N}^{*}\right\}$ and the probability space $(\Omega, \mathcal{P}(\Omega), \mu)$, with the measure $\mu$ defined by $\mu\left(\left\{\zeta_{n}\right\}\right)=\mu_{n}$ and consider the system

$$
\dot{y}_{\zeta}=-\zeta y_{\zeta}+u \quad(\zeta \in \Omega)
$$

According to Proposition 3.1, this system is not exactly simultaneously controllable, although the truncated system in which we consider $\zeta \in\left\{\zeta_{1}, \cdots, \zeta_{N}\right\}$ with the probability measure $\mu^{N}$ given by $\mu^{N}(\{\zeta\})=$ $\frac{\mu(\{\zeta\})}{\mu\left(\left\{\zeta_{1}, \cdots, \zeta_{N}\right\}\right)}$, for $\zeta \in\left\{\zeta_{1}, \cdots, \zeta_{N}\right\}$ is simultaneously controllable, whatever $N \in \mathbb{N}^{*}$ is.

In fact, for this truncated system, the precise values of the measure $\mu^{N}$ are not important since its simultaneous controllability can be understood though the augmented system:

$$
\frac{\mathrm{d}}{\mathrm{d} t}\left(\begin{array}{c}
y_{\zeta_{1}} \\
\vdots \\
y_{\zeta_{N}}
\end{array}\right)=\left(\begin{array}{ccc}
-\zeta_{1} & & 0 \\
& \ddots & \\
0 & & -\zeta_{N}
\end{array}\right)\left(\begin{array}{c}
y_{\zeta_{1}} \\
\vdots \\
y_{\zeta_{N}}
\end{array}\right)+\left(\begin{array}{c}
1 \\
\vdots \\
1
\end{array}\right) u
$$

The Kalman matrix of this system is:

$$
\left(\begin{array}{cccc}
1 & -\zeta_{1} & \cdots & \left(-\zeta_{1}\right)^{N-1} \\
\vdots & \vdots & & \vdots \\
1 & -\zeta_{N} & \cdots & \left(-\zeta_{N}\right)^{N-1}
\end{array}\right)
$$

which is a Vandermonde matrix of determinant $\prod_{1 \leqslant i<j \leqslant N}\left(\zeta_{i}-\zeta_{j}\right) \neq 0$.

Let us now assume that $\sum_{\substack{n \in \mathbb{N}^{*} \\ \zeta_{n} \neq 0}} \frac{1}{\left|\zeta_{n}\right|}<\infty$ and $\mu_{n} \neq 0$ for every $n \in \mathbb{N}^{*}$. Under this assumption, we will prove that this system is approximatively simultaneously controllable in any time $T>0$. In order to prove this result, we have to show the unique continuation property:

$$
\int_{0}^{T}\left|\sum_{n \in \mathbb{N}^{*}} e^{-\zeta_{n}(T-t)} \mathrm{Z}_{n}^{f} \mu_{n}\right|^{2} \mathrm{~d} t=0 \quad \Longrightarrow \quad \forall n \in \mathbb{N}^{*}, \mathrm{z}_{n}^{f}=0 .
$$

This property directly follows from Müntz's theorem, see for instance [14, §12, p. 54].

Proposition 3.1 tells us that it is impossible to build dependent parameter systems which are exactly simultaneously controllable (unless $\operatorname{dim} L^{2}(\Omega, X ; \mu)<\infty$ ). However, as we have seen, the averaged controllability property holds for a variety of models. Consequently, it is natural to look for averaged controls which are optimal in the sense that they minimize the output's variance. This is the core of section 4 . 


\subsection{Momentum approach for simultaneous controllability}

In $\S 3.2$, we gave a necessary and sufficient condition, (3.9), for simultaneous controllability. However, even on simple problems, it is difficult to check whether this condition is satisfied or not. In this paragraph, we present an iterative approach to check whether the observability inequality $(3.9)$ is fulfilled or not. The method presented here can also be seen as an alternative method to the one we proposed in the rest of this paper (see section 4) in order to link averaged controllability to exact simultaneous controllability.

To simplify the notation we define the operator $\mathbb{E} \in \mathcal{L}\left(L^{2}(\Omega, X ; \mu), X\right)$ by:

$$
\mathbb{E}\left(\mathrm{y}_{\zeta}\right)_{\zeta}=\int_{\Omega} \mathrm{y}_{\zeta} \mathrm{d} \mu_{\zeta} \quad\left(\left(\mathrm{y}_{\zeta}\right)_{\zeta} \in L^{2}(\Omega, X ; \mu)\right)
$$

Notice that we have $\mathbb{E}^{*} \mathrm{z}=(\mathrm{z})_{\zeta}$ and $\mathbb{E} \mathbb{E}^{*}=\operatorname{Id}_{X}$.

Let us first remind that proving the averaged controllability property is equivalent to proving that the cost function $J$ defined by (3.5) is coercive and proving the exact simultaneous controllability is equivalent to proving that the cost function $\mathfrak{J}$ defined by $(3.7)$ is coercive. In addition, we have also noticed that we have $J=\mathfrak{J} \circ \mathbb{E}^{*}$, where $\mathbb{E}$ is given by $(3.10)$. Thus, proving that $J$ is coercive means proving that the restriction of $\mathfrak{J}$ to the subset $\mathbb{E}^{*}(X)=\{\zeta \in \Omega \mapsto \mathrm{y} \in X, \mathrm{y} \in X\}$ of $L^{2}(\Omega, X ; \mu)$ is coercive.

Let us also notice that since $L^{2}(\Omega, \mathbb{R} ; \mu)$ is an Hilbert space, one can define an orthonormal basis $\left(\varphi_{i}\right)_{i \in I}$ (with the convention $0 \in I$ and $\varphi_{0}=1$ ) of this space. Based on the above construction of $\mathbb{E}$, we define for every $i \in I$, the operator $\mathbb{E}_{i} \in \mathcal{L}\left(L^{2}(\Omega, X ; \mu), X\right)$ by:

$$
\mathbb{E}_{i}\left(\mathrm{y}_{\zeta}\right)_{\zeta}=\int_{\Omega} \mathrm{y}_{\zeta} \varphi_{i}(\zeta) \mathrm{d} \mu_{\zeta} \quad\left(\left(\mathrm{y}_{\zeta}\right)_{\zeta} \in L^{2}(\Omega, X ; \mu)\right)
$$

so that $L^{2}(\Omega, X ; \mu)=\bigoplus_{i \in I} \mathbb{E}_{i}^{*}(X)$.

Let us assume that $L^{2}(\Omega, \mathbb{R} ; \mu)$ is a separable Hilbert space, that is to say that we can choose $I=\mathbb{N}$ (if $L^{2}(\Omega, \mathbb{R} ; \mu)$ is of infinite dimension) or $I=\{0, \cdots, d\} \subset \mathbb{N}$ (if $L^{2}(\Omega, \mathbb{R} ; \mu)$ is of dimension $d$ ). For every $k \in \mathbb{N}$, we define the finite dimensional subspaces $V_{k}$ of $L^{2}(\Omega, X ; \mu)$ by:

$$
V_{k}=\bigoplus_{\substack{i \in I \\ i \leqslant k}}^{k} \mathbb{E}_{i}^{*}(X) \subset L^{2}(\Omega, X ; \mu)
$$

Let us also define the constant $\hat{c}_{k}(T) \geqslant 0$ by:

$$
\hat{c}_{k}(T)=\inf _{\left(\mathrm{z}_{\zeta}^{f}\right)_{\zeta} \in V_{k} \backslash\{0\}} \frac{\int_{0}^{T}\left\|\int_{\Omega} B_{\zeta}^{*} z_{\zeta}(t) \mathrm{d} \mu_{\zeta}\right\|_{U}^{2} \mathrm{~d} t}{\left\|\mathrm{z}_{\zeta}^{f}\right\|_{L^{2}(\Omega, X: \mu)}^{2}} \quad(k \in \mathbb{N}),
$$

that is to say:

$$
\hat{c}_{k}(T) \int_{\Omega}\left\|\mathrm{z}_{\zeta}^{f}\right\|_{X}^{2} \mathrm{~d} \mu_{\zeta} \leqslant \int_{0}^{T}\left\|\int_{\Omega} B_{\zeta}^{*} z_{\zeta}(t) \mathrm{d} \mu_{\zeta}\right\|_{U}^{2} \mathrm{~d} t \quad\left(k \in \mathbb{N} \quad\left(\mathrm{z}_{\zeta}^{f}\right)_{\zeta} \in V_{k}\right),
$$

with $z_{\zeta}(t)$ the solution of the adjoint problem 3.6 with final condition $z_{\zeta}(T)=\mathrm{z}_{\zeta}^{f} \in V_{k}$. Thus, if $\hat{c}_{k}(T)>0, \mathfrak{J}$ is convex and coercive on $V_{k}$. 
Since $V_{k} \subset V_{k+1}$, the sequence $\left(\hat{c}_{k}(T)\right)_{k \in \mathbb{N}}$ is decreasing. In addition, one can easily convince that if $\lim _{k \rightarrow \infty} \hat{c}_{k}(T)>0$, there exists $\hat{c}(T)>0\left(\hat{c}(T)=\lim _{k \rightarrow \infty} \hat{c}_{k}(T)\right)$ such that:

$$
\hat{c}(T) \int_{\Omega}\left\|\mathrm{z}_{\zeta}^{f}\right\|_{X}^{2} \mathrm{~d} \mu_{\zeta} \leqslant \int_{0}^{T}\left\|\int_{\Omega} B_{\zeta}^{*} z_{\zeta}(t) \mathrm{d} \mu_{\zeta}\right\|_{U}^{2} \mathrm{~d} t \quad\left(\left(\mathrm{z}_{\zeta}^{f}\right)_{\zeta} \in L^{2}(\Omega, X ; \mu)\right) .
$$

That is to say that $\mathfrak{J}$ is convex and coercive on $L^{2}(\Omega, X ; \mu)$ and hence we have exact simultaneous controllability. Moreover, as $k$ goes to infinity, the minimizing family $\left(\mathrm{z}_{k, \zeta}^{f}\right)_{\zeta} \in V_{k}$ of the restriction of $\mathfrak{J}$ to the finite dimensional subspace $V_{k}$ of $L^{2}(\Omega, X ; \mu)$, converges to a minimizing family $\left(\hat{\mathrm{z}}_{\zeta}\right)_{\zeta} \in L^{2}(\Omega, X ; \mu)$ of $\mathfrak{J}$ on $L^{2}(\Omega, X ; \mu)$.

Summarizing the above discussion, leads to the following:

Remark 3.1. Assume that $L^{2}(\Omega, X ; \mu)$ is a separable Hilbert space.

1. If the system (1.1) is approximatively simultaneously controllable, then $\hat{c}_{k}(T) \neq 0$ for every $k \in \mathbb{N}$.

2. If $\lim _{k \rightarrow \infty} \hat{c}_{k}>0$, then the system (1.1) is exactly simultaneously controllable.

3. According to Proposition 3.1, unless $\operatorname{dim} L^{2}(\Omega, X ; \mu)<\infty$, we have $\lim _{k \rightarrow \infty} \hat{c}_{k}=0$.

4. Let us mention that the property (3.14) corresponds to a Kalman rank condition.

More precisely, $\left(\mathrm{z}_{\zeta}^{f}\right)_{\zeta} \in V_{k}$ means there exists $\left(\mathrm{z}^{j, f}\right)_{j=0, \cdots, k} \in X^{k+1}$ such that $\mathrm{z}_{\zeta}^{f}=\sum_{j=0}^{k} \varphi_{j}(\zeta) \mathrm{z}^{j, f}$. Let us then denote by $z_{\zeta}^{j}$ the solution of (3.2) with final condition $\mathrm{z}^{j, f}$. Due to linearity, the solution $z_{\zeta}$ of (3.6) with final condition $\mathrm{z}_{\zeta}^{f}$ is:

$$
z_{\zeta}(t)=\sum_{j=0}^{k} \varphi_{j}(\zeta) z_{\zeta}^{j}(t)=\sum_{j=0}^{k} \varphi_{j}(\zeta) e^{(T-t) A_{\zeta}^{*} \mathrm{Z}^{j, f}}
$$

Finally, since we are in a finite dimensional space the coercive property (3.14) is equivalent to the uniqueness property:

$$
\begin{aligned}
& \int_{\Omega} B_{\zeta}^{*} \sum_{j=0}^{k} \varphi_{j}(\zeta) e^{(T-t) A_{\zeta}^{*} \mathrm{Z}^{j, f} \mathrm{~d} \mu_{\zeta}=0} \quad(t \in[0, T] \quad \text { a.e. }) \\
& \Longrightarrow \quad \mathrm{z}^{j, f}=0 \quad(j \in\{0, \cdots, k\}) .
\end{aligned}
$$

We conclude by time analyticity that (3.14) holds if and only if:

$$
\operatorname{rank}\left[\int_{\Omega}\left(\hat{A}_{\zeta}\right)^{l} \hat{B}_{\zeta} \mathrm{d} \mu_{\zeta}, \quad l \in \mathbb{N}\right]=(k+1) \operatorname{dim} X
$$

where we have defined:

$$
\hat{A}_{\zeta}=\left(\begin{array}{ccc}
A_{\zeta} & & 0 \\
& \ddots & \\
0 & & A_{\zeta}
\end{array}\right) \in \mathcal{L}\left(X^{k+1}\right) \quad \text { and } \quad \hat{B}_{\zeta}=\left(\begin{array}{c}
\varphi_{0}(\zeta) B_{\zeta} \\
\vdots \\
\varphi_{k}(\zeta) B_{\zeta}
\end{array}\right) \in \mathcal{L}\left(U, X^{k+1}\right) \quad(\zeta \in \Omega) .
$$


5. When $\operatorname{Card} \Omega<\infty$, the moments are solution of an ordinary differential equation.

More precisely, consider $\Omega=\{1, \cdots, K\}$ with measure $\mu$ given by $\mu(\{k\})=\theta_{k}$ with $\theta_{k} \in(0,1)$ and $\sum_{k=1}^{K} \theta_{k}=1$. Let us consider an orthonormal basis $\left\{\varphi_{0}, \cdots, \varphi_{K-1}\right\}$ of $L^{2}(\Omega, \mathbb{R} ; \mu)$ (with the convention, $\left.\varphi_{0}(k)=1\right)$. Then the $i^{\text {th }}$-momentum is:

$$
Y_{i}=\sum_{k=1}^{K} \theta_{k} \varphi_{i}(k) y_{k}=\mathbb{M}_{i} \mathbb{I} y \quad(i \in\{0, \cdots, K-1\}),
$$

with:

$$
\begin{aligned}
& y=\left(\begin{array}{c}
y_{1} \\
\vdots \\
y_{K}
\end{array}\right) \in X^{K}, \quad \mathbb{M}_{i}=\left(\varphi_{i}(1) \sqrt{\theta_{1}} \operatorname{Id}_{X} \cdots \varphi_{i}(K) \sqrt{\theta_{K}} \operatorname{Id}_{X}\right) \in \mathcal{L}\left(X^{K}, X\right) \\
& \text { and } \quad \mathbb{I}=\left(\begin{array}{ccc}
\sqrt{\theta_{1}} \operatorname{Id}_{X} & & 0 \\
& \ddots & \\
0 & & \sqrt{\theta_{K}} \operatorname{Id}_{X}
\end{array}\right) \in \mathcal{L}\left(X^{K}\right) \text {. }
\end{aligned}
$$

Thus, setting:

$$
\begin{aligned}
& \mathbb{M}=\left(\begin{array}{c}
\mathbb{M}_{0} \\
\vdots \\
\mathbb{M}_{K-1}
\end{array}\right) \in \mathcal{L}\left(X^{K}\right), \quad \mathbb{A}=\left(\begin{array}{ccc}
A_{1} & & 0 \\
& \ddots & \\
0 & & A_{K}
\end{array}\right) \in \mathcal{L}\left(X^{K}\right) \\
& \text { and } \mathbb{B}=\left(\begin{array}{c}
B_{1} \\
\vdots \\
B_{K}
\end{array}\right) \in \mathcal{L}\left(U, X^{K}\right)
\end{aligned}
$$

the momentums $Y=\left(\begin{array}{c}Y_{0} \\ \vdots \\ Y_{K-1}\end{array}\right)$ satisfies (noticing that $\mathbb{M M}^{\top}=\operatorname{Id}_{X^{K}}$ ):

$$
\dot{Y}=\mathbb{M I A I} \mathbb{I}^{-1} \mathbb{M}^{\top} Y+\mathbb{M I B B} u
$$

Controlling the first $k$ momentums of $\left(y_{k}\right)_{k}$ means controlling the first $k \operatorname{dim} X$ components of $Y$, solution of 3.15 .

Since the basis $\varphi_{0}, \varphi_{1}, \cdots, \varphi_{K-1}$ is free (except $\varphi_{0}=1$ ) one can consider the problem of finding the best possible basis. For instance we can wonder if there exists $\varphi_{1}, \cdots, \varphi_{K-1}$ such that the pair $\left(\mathbb{M I A I} \mathbb{I}^{-1} \mathbb{M}^{\top}, \mathbb{M} \mathbb{B}\right)$ has a normal form (see [15, Proposition 2.2.6]). That is to say find $\varphi_{1}, \cdots, \varphi_{K-1}$ such that $\mathbb{M I A I} \mathbb{I}^{-1} \mathbb{M}^{\top}$ has the structure $\left(\begin{array}{cc}* & * \\ 0 & *\end{array}\right)$ and $\mathbb{M I B}$ the structure $\left(\begin{array}{l}* \\ 0\end{array}\right)$.

\section{A penalty method linking averaged and simultaneous controllability}

As in all this paper, we assume in this section that the assumptions of lemmas 2.1 and 2.2 are satisfied.

In this section, we will present our strategy to link averaged controllability to exact simultaneous controllability. First of all, solving the averaged control problem, can be done with the Hilbert Uniqueness 
Method, that is to say minimize the $L^{2}$-norm of the control with the constraint $\int_{\Omega} y_{\zeta}(T) \mathrm{d} \mu_{\zeta}=\int_{\Omega} \mathrm{y}_{\zeta}^{f} \mathrm{~d} \mu_{\zeta}$. Thus, using Euler-Lagrange formulation (or directly Theorem 3.2), one can see that the averaged control of minimal $L^{2}$-norm is given by (3.4).

In order to reduce the output's variance, one can think to penalise the cost function $\mathcal{J}_{0}$ (given by $\mathcal{J}_{0}(u)=\frac{1}{2}\|u\|_{L^{2}([0, T], U)}^{2}$ with the output's variance, $\int_{\Omega}\left\|y_{\zeta}(T)-\mathrm{y}_{\zeta}^{f}\right\|_{X}^{2} \mathrm{~d} \mu_{\zeta}$. Thus, we introduce the penalized optimization problem:

$$
\begin{array}{ll}
\min & \mathcal{J}_{\kappa}(u):=\frac{1}{2}\|u(t)\|_{L^{2}([0, T], U)}^{2}+\kappa\left\|y_{\zeta}(T ; u)-\mathrm{y}_{\zeta}^{f}\right\|_{L^{2}(\Omega, X ; \mu)}^{2} \quad \quad(\kappa \geqslant 0), \\
& \mathbb{E}\left(y_{\zeta}(T ; u)-\mathrm{y}_{\zeta}^{f}\right)=0 .
\end{array}
$$

where in the above, $y_{\zeta}$ is the solution of 1.1$)$ defined by $(1.2)$ with control $u, L^{2}(\Omega, X ; \mu)$ is the Hilbert space introduced in $(1.3)$ and $\mathbb{E}$ is the expectation defined by $(3.10)$.

Let us give an existence result.

Proposition 4.1. If system (1.1) satisfies the averaged controllability property (1.5) then for every $T>0$, $\left(\mathrm{y}_{\zeta}^{i}\right)_{\zeta},\left(\mathrm{y}_{\zeta}^{f}\right)_{\zeta} \in L^{2}(\Omega, X ; \mu)$ and $\kappa \geqslant 0$, the minimisation problem 4.1) admits one and only one solution $u_{\kappa} \in L^{2}([0, T], U)$.

In addition, the optimal control $u_{\kappa}$ satisfies:

$$
u_{\kappa}(t)=\int_{\Omega} B_{\zeta}^{\star} z_{\zeta}(t) \mathrm{d} \mu_{\zeta} \quad(t \in[0, T])
$$

where, $z_{\zeta}$ is solution of:

$$
\dot{z}_{\zeta}=-A_{\zeta}^{*} z_{\zeta}, \quad z_{\zeta}(T)=\mathrm{z}+\mathrm{y}_{\zeta}^{f}-y_{\zeta}\left(T ; u_{\kappa}\right)
$$

with $\mathrm{z} \in X$ unknown.

Proof. For every $\kappa \geqslant 0$, it is clear that $\mathcal{J}_{\kappa}$ is convex. Since we have assumed that the system (1.1) satisfies the averaged controllability property $(1.5)$, this ensure that the set:

$$
\left\{u \in L^{2}([0, T], U), \mathbb{E}\left(y_{\zeta}(T ; u)-\mathrm{y}_{\zeta}^{f}\right)=0\right\}
$$

is non empty and in addition, this set is a convex and closed set of $L^{2}([0, T], U)$. Moreover, the averaged controllability property ensure that $\mathcal{J}_{0}$ is coercive on this set and consequently $\mathcal{J}_{\kappa}$ is also coercive on this set. Thus, there exists a unique minimizer $u_{\kappa} \in L^{2}([0, T], U)$ for the minimisation problem 4.1.

Let us now prove the optimality conditions. Let us define the Lagrangian of the system:

$$
L(u, \mathrm{z})=\mathcal{J}_{\kappa}(u)+\left\langle\mathrm{z}, \mathbb{E}\left(y_{\zeta}(T ; u)-\mathrm{y}_{\zeta}^{f}\right)\right\rangle_{X} \quad\left(u \in L^{2}([0, T], U), \mathrm{z} \in X\right) .
$$

The optimality conditions are:

$$
\partial_{\mathrm{z}} L=0 \quad \text { and } \quad \partial_{u} L=0 .
$$

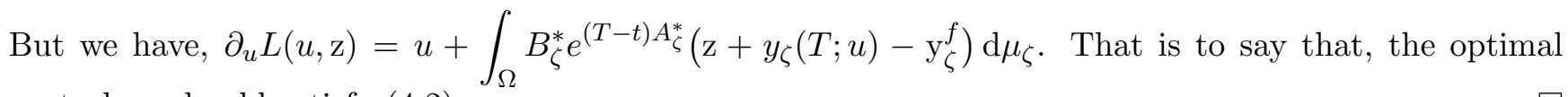
control $u_{\kappa}$ should satisfy 4.2 . 
Of course, we have introduced the cost functions $\mathcal{J}_{\kappa}$ in order to pass to the limit $\kappa \rightarrow \infty$. Let us first state a trivial statement:

Lemma 4.1. Set $T>0$ and assume the system (1.1) is controllable in average.

For every $\kappa \geqslant 0$, let us define $u_{\kappa}$ the minimum of $\mathcal{J}_{\kappa}$ under the constraint $\mathbb{E}\left(y_{\zeta}\left(T, u_{\kappa}\right)-\mathrm{y}_{\zeta}^{f}\right)=0$.

Then, we have:

$$
\begin{aligned}
\left\|u_{\kappa}\right\|_{L^{2}([0, T], U)} \leqslant\left\|u_{\kappa+\varepsilon}\right\|_{L^{2}([0, T], U)} \quad \text { and } \\
\left\|y_{\zeta}\left(T ; u_{\kappa}\right)-\mathrm{y}_{\zeta}^{f}\right\|_{L^{2}(\Omega, X ; \mu)} \geqslant\left\|y_{\zeta}\left(T ; u_{\kappa+\varepsilon}\right)-\mathrm{y}_{\zeta}^{f}\right\|_{L^{2}(\Omega, X ; \mu)} \quad(\kappa, \varepsilon \geqslant 0) .
\end{aligned}
$$

In addition, for every $\kappa \geqslant 0$, we have:

$$
\begin{array}{r}
\left\|y_{\zeta}\left(T, u_{\kappa}\right)-\mathrm{y}_{\zeta}^{f}\right\|_{L^{2}(\Omega, X ; \mu)} \\
=\min \left\{\left\|y_{\zeta}(T ; u)-\mathrm{y}_{\zeta}^{f}\right\|_{L^{2}(\Omega, X ; \mu)}, u \in L^{2}([0, T], U), \quad\|u\|_{L^{2}([0, T], U)} \leqslant\left\|u_{\kappa}\right\|_{L^{2}([0, T], U)}\right. \\
\left.\quad \text { and } \mathbb{E}\left(y_{\zeta}(T ; u)-\mathrm{y}_{\zeta}^{f}\right)=0\right\} .
\end{array}
$$

Proof. It remains clear that for every $\kappa, \varepsilon \geqslant 0$, we have:

$$
\mathcal{J}_{\kappa}\left(u_{\kappa}\right) \leqslant \mathcal{J}_{\kappa}\left(u_{\kappa+\varepsilon}\right) \leqslant \mathcal{J}_{\kappa+\varepsilon}\left(u_{\kappa+\varepsilon}\right) \leqslant \mathcal{J}_{\kappa+\varepsilon}\left(u_{\kappa}\right) .
$$

Thus from, $\mathcal{J}_{\kappa}\left(u_{\kappa}\right)+\mathcal{J}_{\kappa+\varepsilon}\left(u_{\kappa+\varepsilon}\right) \leqslant \mathcal{J}_{\kappa}\left(u_{\kappa+\varepsilon}\right)+\mathcal{J}_{\kappa+\varepsilon}\left(u_{\kappa}\right)$, it is easy to see that $\left(\left\|y_{\zeta}\left(T ; u_{\kappa}\right)-\mathrm{y}_{\zeta}^{f}\right\|_{L^{2}(\Omega, X ; \mu)}\right)_{\kappa \geqslant 0}$ is decreasing and then, form $\mathcal{J}_{\kappa}\left(u_{\kappa}\right) \leqslant \mathcal{J}_{\kappa}\left(u_{\kappa+\varepsilon}\right)$, we obtain that $\left(\left\|u_{\kappa}\right\|_{L^{2}([0, T], U)}\right)_{\kappa \geqslant 0}$ is increasing.

Let us now prove 4.3$)$. To this end, we assume by contradiction that there exists $u \in L^{2}([0, T], U)$ such that:

$$
\begin{aligned}
\|u\|_{L^{2}([0, T], U)} \leqslant\left\|u_{\kappa}\right\|_{L^{2}([0, T], U)}, \quad \mathbb{E}\left(y_{\zeta}(T ; u)-\mathrm{y}_{\zeta}^{f}\right)=0 \\
\quad \text { and }\left\|y_{\zeta}(T ; u)-\mathrm{y}_{\zeta}^{f}\right\|_{L^{2}(\Omega, X ; \mu)}<\left\|y_{\zeta}\left(T ; u_{\kappa}\right)-\mathrm{y}_{\zeta}^{f}\right\|_{L^{2}(\Omega, X ; \mu)} .
\end{aligned}
$$

Then we have $\mathcal{J}_{\kappa}(u)<\mathcal{J}_{\kappa}\left(u_{\kappa}\right)$ which is in contradiction with $u_{\kappa}$ minimize $\mathcal{J}_{\kappa}$.

Various situations could hold as $\kappa \rightarrow \infty$. These different situations, reported on Table 1, are given by the following theorem.

Theorem 4.1. Set $T>0$ and assume that the system (1.1) in controllable in average in time $T$. For every $\kappa \geqslant 0$, let us define $u_{\kappa}$ the minimum of $\mathcal{J}_{\kappa}$ under the constraint $\mathbb{E}\left(y_{\zeta}\left(T, u_{\kappa}\right)-\mathrm{y}_{\zeta}^{f}\right)=0$. Define $\left(\mathrm{y}_{\zeta}^{\star}\right)_{\zeta} \in L^{2}(\Omega, X ; \mu)$ as the minimizer of:

$$
\begin{array}{ll}
\min & \left\|\mathrm{y}_{\zeta}-\mathrm{y}_{\zeta}^{f}\right\|_{L^{2}(\Omega, X ; \mu)} \\
& \left(\mathrm{y}_{\zeta}\right)_{\zeta} \in \overline{\left\{y_{\zeta}(T ; u), u \in L^{2}([0, T], U)\right\}} \\
& \mathbb{E}\left(\mathrm{y}_{\zeta}\right)_{\zeta}=\mathbb{E}\left(\mathrm{y}_{\zeta}^{f}\right)_{\zeta} .
\end{array}
$$

Then, the following alternative holds: 
- If $\left(\left\|u_{\kappa}\right\|_{L^{2}([0, T], U)}\right)_{\kappa \geqslant 0}$ is bounded, then $\left(u_{\kappa}\right)_{\kappa}$ converges to a control which steers exactly $\mathrm{y}_{\zeta}^{i}$ to $\mathrm{y}_{\zeta}^{\star}$ and realises the minimum of:

$$
\begin{array}{cl}
\min & \frac{1}{2}\|u\|_{L^{2}([0, T], U)}^{2} \\
\mid & \left\|y_{\zeta}(T ; u)-\mathrm{y}_{\zeta}^{\star}\right\|_{L^{2}(\Omega, X ; \mu)}=0
\end{array}
$$

- If $\left(\left\|u_{\kappa}\right\|_{L^{2}([0, T], U)}\right)_{\kappa \geqslant 0}$ is unbounded, then $\mathrm{y}_{\zeta}^{i}$ can be approximatively steered to $\mathrm{y}_{\zeta}^{\star}$.

In addition, if $\lim _{\kappa \rightarrow \infty}\left(\left\|y_{\zeta}\left(T ; u_{\kappa}\right)-\mathrm{y}_{\zeta}^{f}\right\|_{L^{2}(\Omega, X ; \mu)}\right)=0$, then we have $\mathrm{y}_{\zeta}^{\star}=\mathrm{y}_{\zeta}^{f}$.

Proof. Without loss of generality, we can assume that $\mathrm{y}_{\zeta}^{i}=0$.

Let us first notice that $\left(\mathrm{y}_{\zeta}^{\star}\right)_{\zeta} \in L^{2}(\Omega, X ; \mu)$ is well defined. In fact, $\left(\mathrm{y}_{\zeta}^{\star}\right)_{\zeta}$ is the orthogonal projection of $\left(\mathrm{y}_{\zeta}^{f}\right)_{\zeta}$ in $L^{2}(\Omega, X ; \mu)$ on the closed vector space $\overline{\left\{y_{\zeta}(T ; u), u \in L^{2}([0, T], U)\right\}} \cap\left\{\left(\mathrm{y}_{\zeta}\right)_{\zeta}, \mathbb{E}\left(\mathrm{y}_{\zeta}\right)_{\zeta}=\mathbb{E}\left(\mathrm{y}_{\zeta}^{f}\right)_{\zeta}\right\}$.

- Let us assume $\left(\left\|u_{\kappa}\right\|_{L^{2}([0, T], U)}\right)_{\kappa \geqslant 0}$ bounded.

From Lemma 4.1, the sequence $\left(\left\|u_{\kappa}\right\|_{L^{2}([0, T], U)}\right)_{\kappa \geqslant 0}$ is increasing, hence there exists $u_{\infty} \in L^{2}([0, T], U)$ such that up to a subsequence, $\left(u_{\kappa}\right)_{\kappa \geqslant 0}$ is weakly convergent to $u_{\infty}$ and in addition, we have:

$$
\left\|u_{\infty}\right\|_{L^{2}([0, T], U)} \leqslant \lim _{\kappa \rightarrow \infty}\left\|u_{\kappa}\right\|_{L^{2}([0, T], U)} .
$$

Since $\left(u_{\kappa}\right)_{\kappa \geqslant 0}$ is weakly convergent to $u_{\infty}$, it is easy to obtain that $\left(\left(y_{\zeta}\left(T ; u_{\kappa}\right)\right)_{\zeta}\right)_{\kappa \geqslant 0}$ is weakly convergent to $\left(y_{\zeta}\left(T ; u_{\infty}\right)\right)_{\zeta} \in L^{2}(\Omega, X ; \mu)$. Hence,

$$
\mathbb{E}\left(y_{\zeta}\left(T ; u_{\infty}\right)\right)_{\zeta}=\mathbb{E}\left(y_{\zeta}^{f}\right)_{\zeta} \quad \text { and } \quad\left\|y_{\zeta}\left(T ; u_{\infty}\right)-\mathrm{y}_{\zeta}^{f}\right\|_{L^{2}(\Omega, X ; \mu)} \leqslant \liminf _{\kappa \rightarrow \infty}\left\|y_{\zeta}\left(T ; u_{\kappa}\right)-\mathrm{y}_{\zeta}^{f}\right\|_{L^{2}(\Omega, X ; \mu)} .
$$

In addition, from Lemma 4.1, the sequence $\left(\left\|y_{\zeta}\left(T ; u_{\kappa}\right)-y_{\zeta}^{f}\right\|_{L^{2}(\Omega, X ; \mu)}\right)_{\kappa}$ is decreasing thus, we have:

$$
\left\|y_{\zeta}\left(T ; u_{\infty}\right)-\mathrm{y}_{\zeta}^{f}\right\|_{L^{2}(\Omega, X ; \mu)} \leqslant\left\|y_{\zeta}\left(T ; u_{\kappa}\right)-\mathrm{y}_{\zeta}^{f}\right\|_{L^{2}(\Omega, X ; \mu)} \quad(\kappa>0)
$$

and hence, from relation (4.3) of Lemma 4.1. we obtain $\left\|u_{\infty}\right\|_{L^{2}([0, T], U)} \geqslant\left\|u_{\kappa}\right\|_{L^{2}([0, T], U)}$ that is to say, $\left\|u_{\infty}\right\|_{L^{2}([0, T], U)}=\lim _{\kappa \rightarrow \infty}\left\|u_{\kappa}\right\|_{L^{2}([0, T], U)}$ and (up to a subsequence, $\left(u_{\kappa}\right)_{\kappa}$ is strongly convergent to $u_{\infty}$ in $L^{2}([0, T], U)$. Consequently, $\left(\left(y_{\zeta}\left(T ; u_{\kappa}\right)\right)_{\zeta}\right)_{\kappa}$ is strongly convergent to $\left(y_{\zeta}\left(T ; u_{\infty}\right)\right)_{\zeta}$ in $L^{2}(\Omega, X ; \mu)$.

Let us now prove that $y_{\zeta}\left(T, u_{\infty}\right)=\mathrm{y}_{\zeta}^{\star}$. Assume by contradiction that it is not the case. That is to say there exists $\bar{u} \in L^{2}([0, T], U)$ such that:

$$
\mathbb{E}\left(y_{\zeta}(T ; \bar{u})\right)_{\zeta}=\mathbb{E}\left(\mathrm{y}_{\zeta}^{f}\right)_{\zeta} \quad \text { and } \quad\left\|y_{\zeta}(T ; \bar{u})-\mathrm{y}_{\zeta}^{f}\right\|_{L^{2}(\Omega, X ; \mu)}<\left\|y_{\zeta}\left(T, u_{\infty}\right)-\mathrm{y}_{\zeta}^{f}\right\|_{L^{2}(\Omega, X ; \mu)} .
$$

But, we have $\mathcal{J}_{\kappa}\left(u_{\kappa}\right) \leqslant \mathcal{J}_{\kappa}(\bar{u})$ for every $\kappa \geqslant 0$, i.e.:

$$
\begin{aligned}
\frac{1}{2 \kappa}\left(\|\bar{u}\|_{L^{2}([0, T], U)}^{2}-\left\|u_{\kappa}\right\|_{L^{2}([0, T], U)}\right) & \\
& \geqslant\left\|y_{\zeta}\left(T ; u_{\kappa}\right)-\mathrm{y}_{\zeta}^{f}\right\|_{L^{2}(\Omega, X ; \mu)}^{2}-\left\|y_{\zeta}(T ; \bar{u})-\mathrm{y}_{\zeta}^{f}\right\|_{L^{2}(\Omega, X ; \mu)}^{2} \quad(\kappa>0) .
\end{aligned}
$$

Taking the limit $\kappa \rightarrow \infty$ comes the contradiction:

$$
\left\|y_{\zeta}(T ; \bar{u})-\mathrm{y}_{\zeta}^{f}\right\|_{L^{2}(\Omega, X ; \mu)}^{2} \geqslant\left\|y_{\zeta}\left(T ; u_{\infty}\right)-\mathrm{y}_{\zeta}^{f}\right\|_{L^{2}(\Omega, X ; \mu)}^{2} .
$$


Let us now show that $\left(u_{\kappa}\right)_{\kappa}$ is convergent to $u_{\infty}$ (that is to say that we do not need the subsequence extraction procedure). This follows form the fact that any convergent subsequence of $\left(u_{\kappa}\right)_{\kappa}$ is convergent to a minimizer of (4.5). But it is trivial that the minimization problem (4.5) has at most one minimizer.

Finally, it remains clear that $\lim _{\kappa \rightarrow \infty}\left\|y_{\zeta}\left(T ; u_{\kappa}\right)-\mathrm{y}_{\zeta}^{f}\right\|_{L^{2}(\Omega, X ; \mu)}=0$ is equivalent to $\mathrm{y}_{\zeta}^{\star}=\mathrm{y}_{\zeta}^{f}$.

- Let us assume that $\left(\left\|u_{\kappa}\right\|_{L^{2}([0, T], U)}\right)_{\kappa \geqslant 0}$ is not bounded.

The results of this point are direct consequences of 4.3 given in Lemma 4.1.

If the system (1.1) is simultaneously controllable, the convergence rates to the simultaneous control and the one of the variance to 0 are linked.

Proposition 4.2. Assume system (1.1) is exactly simultaneously controllable in time $T>0$. Let $u_{\infty} \in$ $L^{2}([0, T], U)$ be the exact simultaneous control of minimal norm steering $\mathrm{y}_{\zeta}^{i}$ to $\mathrm{y}_{\zeta}^{f}$ and let $u_{\kappa} \in L^{2}([0, T], U)$ be the minimizer of 4.1).

Then, $\left(u_{\kappa}\right)_{\kappa \geqslant 0}$ is strongly convergent to $u_{\infty}$ and, in addition,

$$
\left\|y_{\zeta}\left(T, u_{\kappa}\right)-\mathrm{y}_{\zeta}^{f}\right\|_{L^{2}(\Omega, X ; \mu)}^{2} \leqslant\left\|u_{\infty}\right\|_{L^{2}([0, T], U)} \kappa^{-1}\left\|u_{\kappa}-u_{\infty}\right\|_{L^{2}([0, T], U)} .
$$

Proof. First of all, $\left(u_{\kappa}\right)_{\kappa}$ is strongly convergent to $u_{\infty}$ is a consequence of Theorem 4.1.

Let us now prove (4.6). First of all, changing $\mathrm{y}_{\zeta}^{f}$ in $\mathrm{y}^{f}-e^{T A_{\zeta}} \mathrm{y}_{\zeta}^{i}$, we can assume without loss of generality that $\mathrm{y}_{\zeta}^{i}=0$.

Set $u_{\kappa}=u_{\infty}+v_{\kappa}$, then $v_{\kappa}$ is a minimizer of:

$$
\begin{aligned}
\min & \mathcal{G}_{\kappa}(v)=\frac{1}{2}\|v\|_{L^{2}([0, T], U)}^{2}+\left\langle v, u_{\infty}\right\rangle_{L^{2}([0, T], U)}+\kappa \int_{\Omega} \| \int_{0}^{T} e^{(T-t) A_{\zeta} B_{\zeta} v(t) \mathrm{d} t \|_{X}^{2} \mathrm{~d} \mu_{\zeta}} \\
& \mathbb{E}\left(\int_{0}^{T} e^{\left.(T-t) A_{\zeta} B_{\zeta} v(t) \mathrm{d} t\right)=0 .}\right.
\end{aligned}
$$

We have:

$$
\mathcal{G}_{\kappa}\left(v_{\kappa}\right) \leqslant \mathcal{G}_{\kappa}(0)=0
$$

Thus, for every $\kappa \geqslant 0$,

$$
\begin{aligned}
\kappa \int_{\Omega}\left\|\int_{0}^{T} e^{(T-t) A_{\zeta}} B_{\zeta} v_{\kappa}(t) \mathrm{d} t\right\|^{2} \mathrm{~d} \mu_{\zeta} \leqslant \frac{1}{2}\left\|v_{\kappa}\right\|_{L^{2}([0, T], U)}^{2}+\kappa \int_{\Omega}\left\|\int_{0}^{T} e^{(T-t) A_{\zeta}} B_{\zeta} v_{\kappa}(t) \mathrm{d} t\right\|^{2} \mathrm{~d} \mu_{\zeta} \\
\leqslant-\left\langle v_{\kappa}, u_{\infty}\right\rangle_{L^{2}([0, T], U)} \leqslant\left\|v_{\kappa}\right\|_{L^{2}([0, T], U)}\left\|u_{\infty}\right\|_{L^{2}([0, T], U)} .
\end{aligned}
$$

This ends the proof using $\int_{0}^{T} e^{(T-t) A_{\zeta}} B_{\zeta} v_{\kappa}(t) \mathrm{d} t=y_{\zeta}\left(T ; u_{\kappa}\right)-y_{\zeta}\left(t ; u_{\infty}\right)=y_{\zeta}\left(T ; u_{\kappa}\right)-y_{\zeta}^{f}$.

Let us now give the consequences of Theorem 4.1 in the case where the cardinal of $\Omega$ is finite.

Corollary 4.1. Assume $L^{2}(\Omega, X ; \mu)$ is of finite dimension.

Then the sequence of minimizers $\left(\hat{u}_{\kappa}\right)_{\kappa}$ of the optimisation problem (4.1) is strongly convergent to an element $\hat{u}_{\infty} \in L^{2}([0, T], U)$ satisfying the minimisation problem:

$$
\begin{array}{cl}
\min & \frac{1}{2}\|u\|_{L^{2}([0, T], U)}^{2} \\
& y_{\zeta}(T)=\mathrm{y}_{\zeta}^{\star}
\end{array} \quad(\zeta \in \Omega \quad \mu-\text { a.e. }),
$$

where $\mathrm{y}_{\zeta}^{\star}$, defined by Theorem 4.1, is the minimizer of 4.4. 
A graphical interpretation of this result is given on Figure 2.

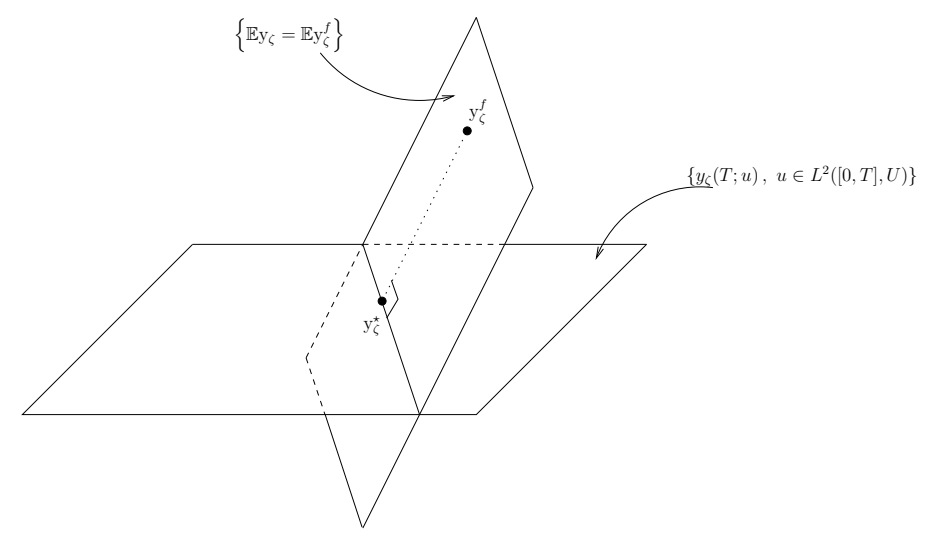

Figure 2: Under the assumptions of Corollary 4.1, at the limit $\kappa \rightarrow \infty$, the emergent control will be a control steering $\left(\mathrm{y}_{\zeta}^{i}\right)_{\zeta}$ to $\left(\mathrm{y}_{\zeta}^{\star}\right)_{\zeta}$.

Proof. Let us use the notations introduced in Theorem 4.1. Since $L^{2}(\Omega, X ; \mu)$ is a finite dimensional space, $\left\{y_{\zeta}(T ; u), u \in L^{2}([0, T], U)\right\} \cap\left\{\left(\mathrm{y}_{\zeta}\right)_{\zeta}, \mathbb{E}\left(\mathrm{y}_{\zeta}\right)_{\zeta}=\mathbb{E}\left(\mathrm{y}_{\zeta}^{f}\right)_{\zeta}\right\}$ is a closed affine subspace of $L^{2}(\Omega, X ; \mu)$. Consequently, there exists $u^{\star} \in L^{2}([0, T], U)$ such that $\mathrm{y}_{\zeta}^{\star}=y_{\zeta}\left(T ; u^{\star}\right)$.

Example 4.1. This example illustrates the result of Corollary 4.1 in the exact simultaneous controllability case.

Consider the probability space $(\Omega, \mathcal{P}(\Omega), \mu)$ with $\Omega=\{1,2\}$ and $\mu(\{1\})=\mu(\{2\})=\frac{1}{2}$. The parameter dependent system under consideration is:

$$
\dot{y}_{\zeta}=\zeta A y_{\zeta}+B u \quad y_{\zeta}(0)=y^{i} \quad(\zeta \in\{1,2\}),
$$

with $A=\left(\begin{array}{cc}0 & -1 \\ 1 & 0\end{array}\right), B=\left(\begin{array}{l}1 \\ 0\end{array}\right)$ and $\mathrm{y}^{i}=\left(\begin{array}{l}1 \\ 1\end{array}\right)$.

We fix the final target $\mathrm{y}^{f}$ to $(0,0)^{\top}$ and the final time $T$ to 1 .

The corresponding augmented system is:

$$
\dot{\boldsymbol{y}}=\mathbf{A} \boldsymbol{y}+\mathbf{B} u \quad \boldsymbol{y}(0)=\mathbf{y}^{i},
$$

with $\mathbf{A}=\left(\begin{array}{cc}A & 0 \\ 0 & 2 A\end{array}\right)=\left(\begin{array}{cccc}0 & -1 & 0 & 0 \\ 1 & 0 & 0 & 0 \\ 0 & 0 & 0 & -2 \\ 0 & 0 & 2 & 0\end{array}\right), \mathbf{B}=\left(\begin{array}{l}B \\ B\end{array}\right)=\left(\begin{array}{l}1 \\ 0 \\ 1 \\ 0\end{array}\right)$ and $\mathbf{y}^{i}=\left(\begin{array}{c}\mathrm{y}^{i} \\ \mathrm{y}^{i}\end{array}\right)=\left(\begin{array}{l}1 \\ 1 \\ 1 \\ 1\end{array}\right)$.

Using the Kalman rank condition, it is easy to see that this augmented system is controllable (in the classical sense) and controlling the average means controlling $\frac{1}{2}\left(\boldsymbol{y}_{1}+\boldsymbol{y}_{3}, \boldsymbol{y}_{2}+\boldsymbol{y}_{4}\right)^{\top}$.

On figures 3. 4 and 5, we plot the numerical results dealing with the averaged control, the exact simultaneous control and the solution of the penalisation problem, when letting the parameter $\kappa$ growing. 


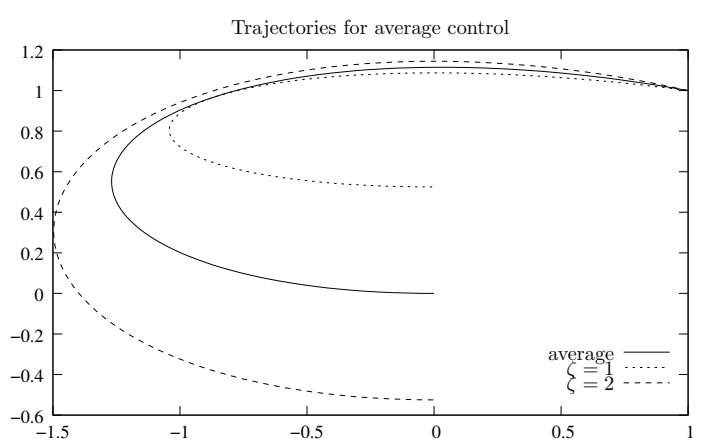

(a) Controlled trajectories in the phase plan using the averaged control. The variance at final time is $2.75 e-01$.

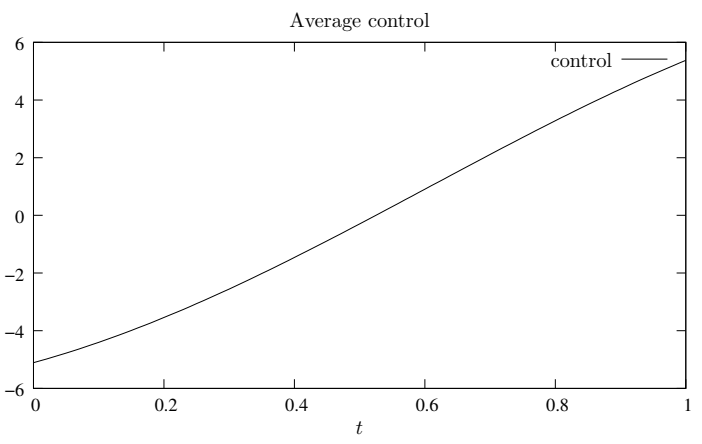

(b) Averaged control, the norm of the control is 3.19 .

Figure 3: On left, we plotted the trajectories obtained by the averaged control (right) which is of minimal $L^{2}$-norm.

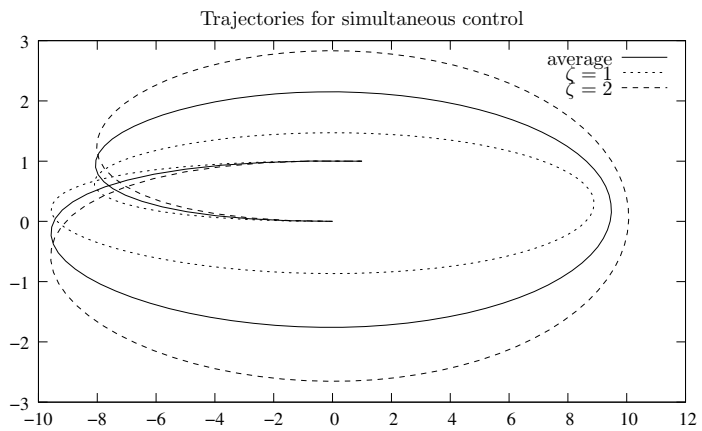

(a) Controlled trajectories in the phase plan using the simultaneous control.

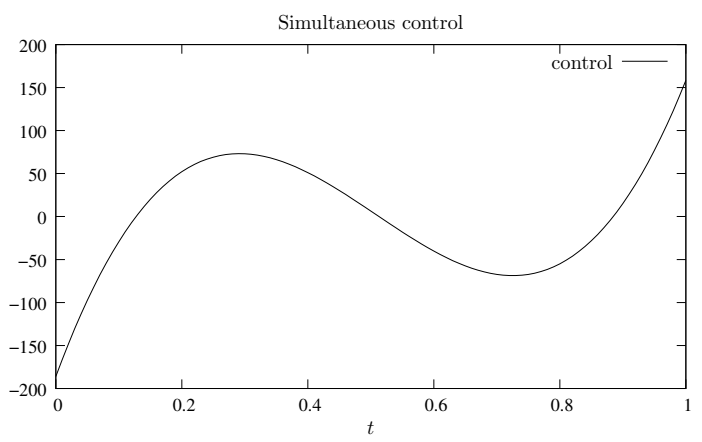

(b) Simultaneous control, the norm of the control is $6.34 \mathrm{e}+01$.

Figure 4: On left, we plotted the trajectories obtained by the simultaneous control (right) which is of minimal $L^{2}$-norm.

Example 4.2. This example illustrates the result of Corollary 4.1 when there is no simultaneous controllability. For this example, we consider again the probability space $\Omega=\{1,2\}$ and the probability density $\mu$ given by $\mu(\{1\})=\mu(\{2\})=\frac{1}{2}$. The parameter dependent system under consideration is:

$$
\begin{aligned}
\dot{y}_{\zeta}=A_{\zeta} y_{\zeta}+B u \quad y_{\zeta}(0)=\mathrm{y}^{i}, & \left\{\begin{array}{l}
\left(\begin{array}{cc}
0 & -1 \\
1 & 0
\end{array}\right) \quad \text { if } \zeta=1, \\
\text { with } B=\left(\begin{array}{l}
1 \\
0
\end{array}\right), \mathrm{y}^{i}=\left(\begin{array}{l}
1 \\
1
\end{array}\right) \text { and } A_{\zeta}=\left\{\begin{array}{ll}
1 & 0 \\
0 & 1
\end{array}\right) \quad \text { if } \zeta=2 .
\end{array}\right.
\end{aligned}
$$

Using the Kalman rank condition, introduced by E. Zuazua (see Theorem 3.1), one can see that this system is controllable in average. On the other hand, the simultaneous controllability of this system reduce to prove the classical controllability of the augmented system:

$$
\dot{\boldsymbol{y}}=\mathbf{A} \boldsymbol{y}+\mathbf{B} u
$$




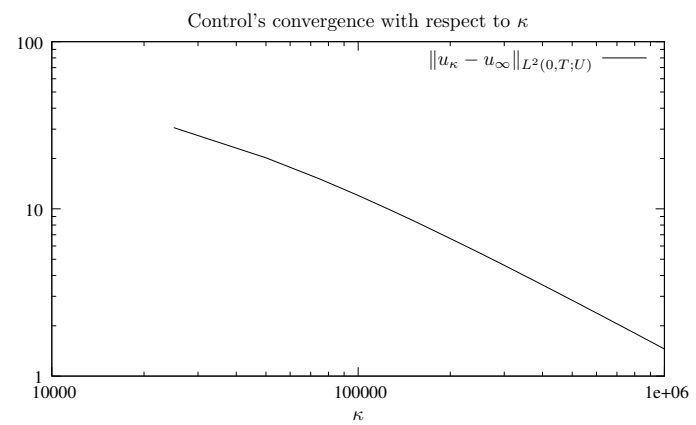

(a) Plot of the $L^{2}$-distance between the exact simultaneous control and the optimal control of the minimisation problem indexed with $\kappa$. This distance behaves as $C \kappa^{-\alpha}$ with $\alpha \simeq 0.98$.

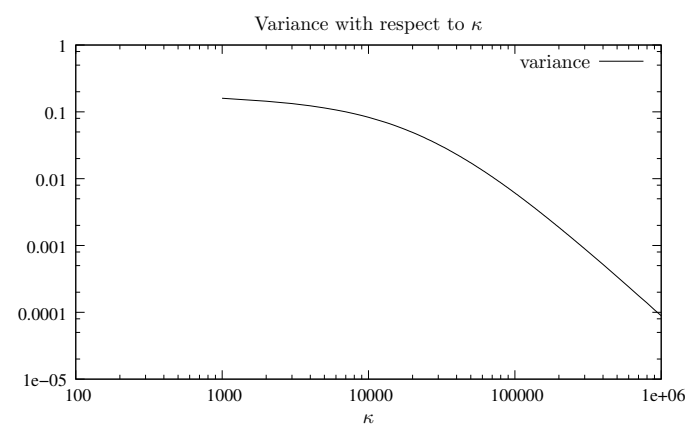

(b) Plot of the variance at final state $\left(\int\left\|y_{\zeta}(T)-\mathrm{y}^{f}\right\|_{X}^{2} \mathrm{~d} \mu_{\zeta}\right)$ with respect to $\kappa$. The variance behaves as $C \kappa^{-\alpha}$ with $\alpha \simeq 1.95$.

Figure 5: Plots in $\log -\log$ scale of the $L^{2}$-distance between the solution of the optimal control with parameter $\kappa$ and the exact simultaneous control (left) and of the variance at final state (right) as $\kappa$ grows. The decay rates obtained are coherent with the results of Proposition 4.2.

with $\mathbf{A}=\left(\begin{array}{cc}A_{1} & 0 \\ 0 & A_{2}\end{array}\right)=\left(\begin{array}{cccc}0 & -1 & 0 & 0 \\ 1 & 0 & 0 & 0 \\ 0 & 0 & 1 & 0 \\ 0 & 0 & 0 & 1\end{array}\right)$ and $\mathbf{B}=\left(\begin{array}{l}B \\ B\end{array}\right)=\left(\begin{array}{l}1 \\ 0 \\ 1 \\ 0\end{array}\right)$.

One can easily see that $\operatorname{rank}\left[\mathbf{B}, \mathbf{A B}, \mathbf{A}^{2} \mathbf{B}, \mathbf{A}^{3} \mathbf{B}\right]=3<4$ and hence, the Kalman rank condition is not satisfied.

On figures 6. 7 and 8, we present the numerical results for this system. As in Example 4.1, the final time $T$ is set to 1 and the target $\mathrm{y}^{f}$ is $(0,0)^{\top}$.

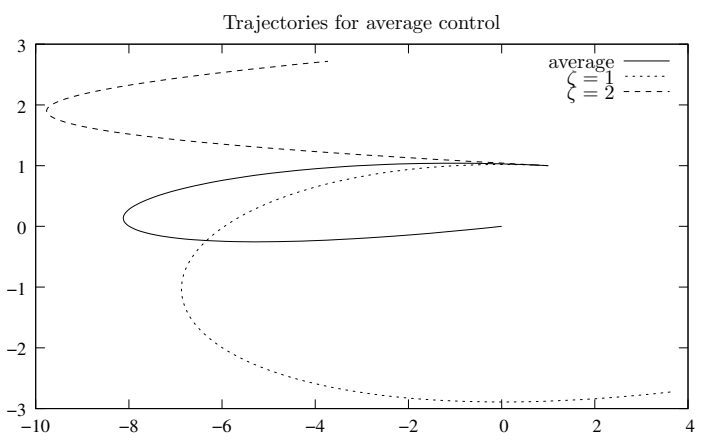

(a) Controlled trajectories in the phase plan using the averaged control. The variance at final time is $2.13 e+01$.

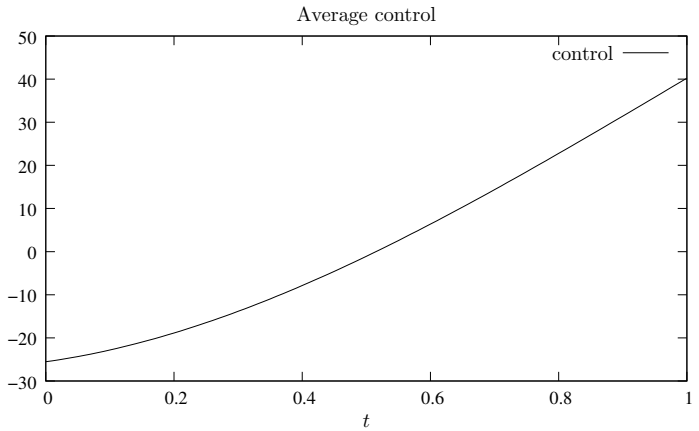

(b) Averaged control, the $L^{2}$-norm of the control is $1.99 \mathrm{e}+01$.

Figure 6: On left, we plotted the trajectories obtained by the averaged control (right) which is of minimal $L^{2}$-norm. 


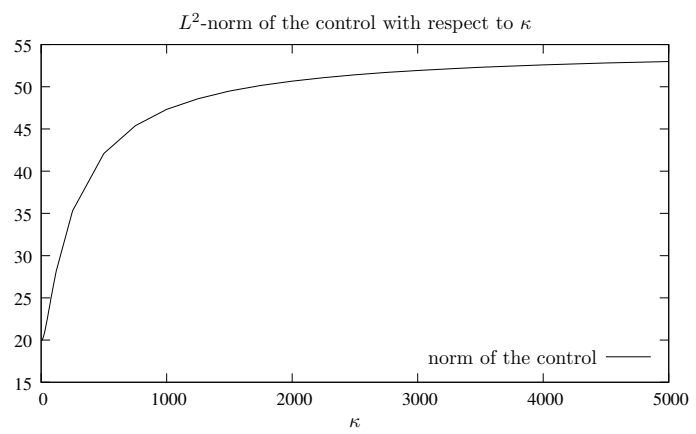

(a) Plot of the norm of the control with respect to $\kappa$.

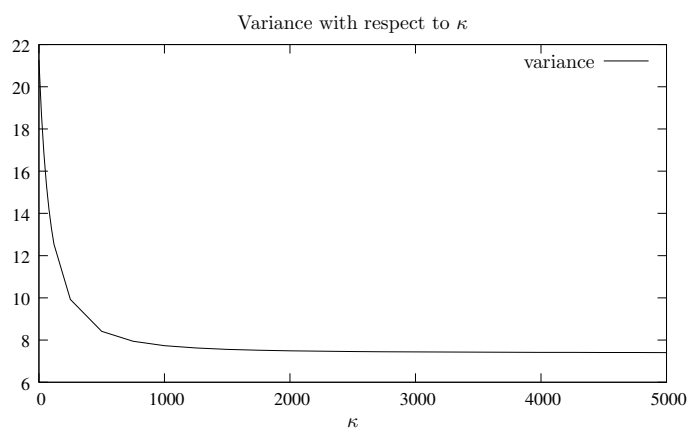

(b) Plot of the variance at final state with respect to $\kappa$.

Figure 7: Plots of the norm of the control (left) and of the variance at final state (right) as $\kappa$ grows.

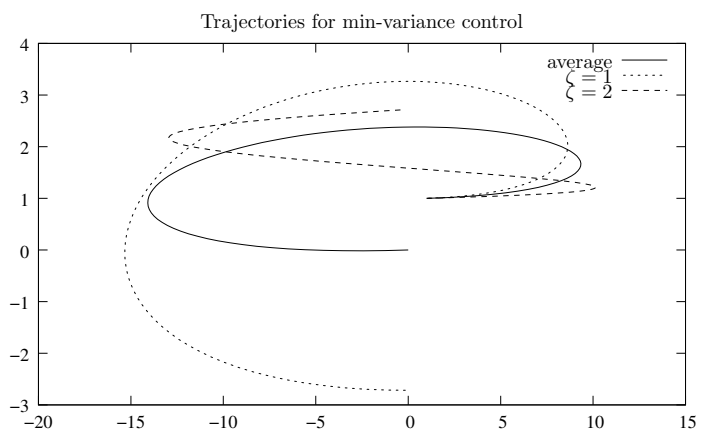

(a) Controlled trajectories in the phase plan using the optimal control for $\kappa=5.10^{3}$. The variance at final time is 7.41 .

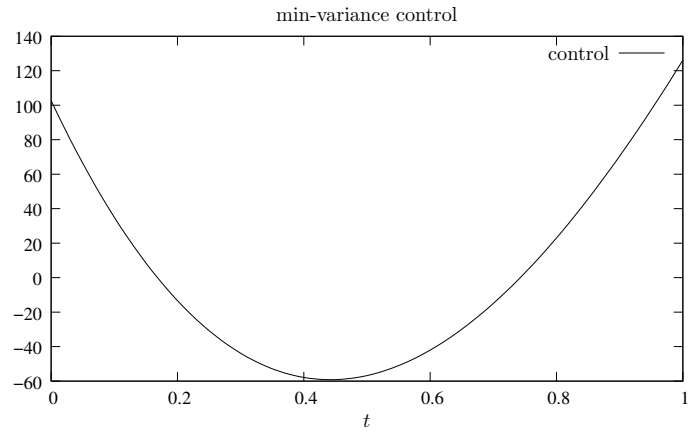

(b) Optimal control for $\kappa=5.10^{3}$ its $L^{2}$-norm is $5.30 \mathrm{e}+01$.

Figure 8: On left, we plotted the trajectories obtained by the optimal control (right) for $\kappa=5.10^{3}$.

\section{Numerical realisation when $\operatorname{Card} \Omega$ is infinite}

In this section we will study the discrete event case $\left(\Omega=\mathbb{N}^{*}\right)$.

For this case, we consider the probability space $\left(\mathbb{N}^{*}, \mathcal{P}\left(\mathbb{N}^{*}\right), \mu\right)$. A natural way to deal with this problem is to truncate it. More precisely, instead of considering the probability space $\left(\mathbb{N}^{*}, \mathcal{P}\left(\mathbb{N}^{*}\right), \mu\right)$, we consider the probability space $\left(\mathbb{N}^{*}, \mathcal{P}\left(\mathbb{N}^{*}\right), \chi_{Z} \mu\right)$ with the measure $\chi_{Z} \mu$ given by

$$
\chi_{Z} \mu(\{\zeta\})=\left\{\begin{array}{ll}
\frac{\mu(\{\zeta\})}{\mu(\{1, \cdots, Z\})} & \text { if } \zeta \leqslant Z, \\
0 & \text { otherwise, }
\end{array} \quad\left(Z \in \mathbb{N}^{*}, \zeta \in \mathbb{N}^{*}\right),\right.
$$

for $Z \in \mathbb{N}^{*}$ large enough so that $\mu(\{1, \cdots, Z\})>0$.

Since our penalisation procedure needs the system $\dot{y}_{\zeta}=A_{\zeta} y_{\zeta}+B_{\zeta} u$ to be controllable in average the first question we should answer is whether this averaged controllability property is stable or not through the truncation procedure.

Proposition 5.1. Assume the system (1.1) is controllable in average for the measure $\mu$.

Then there exists $Z_{0} \in \mathbb{N}^{*}$ such that for every $Z \geqslant Z_{0}$, this system is controllable in average for the measure $\chi_{Z} \mu$ given by (5.1). 
Let us also notice that this truncation procedure does not affect the simultaneous controllability property for $Z$ large enough.

Proposition 5.2. Assume the system (1.1) is approximatively simultaneously controllable for the measure $\mu$.

Then for every $Z \in \mathbb{N}^{*}$ such that $\mu(\{1, \cdots, Z\})>0$, this system is simultaneously controllable for the measure $\chi_{Z} \mu$ given by (5.1).

Remark 5.1. Notice that by truncation, one can lose the averaged controllability property. This is for instance the case of the system considered in Example 4.2.

In opposition the simultaneous controllability property cannot be lost by truncation. This is natural since if the system in simultaneously controllable, all the events $y_{1}, \cdots, y_{Z}$ can be exactly controlled. Of course, as Example 3.1 shows, the reverse property does not hold.

Consequently, if a system is simultaneously controllable, then it is controllable in average and each of its truncation is controllable in average.

The reverse property does not hold, i.e. there exist systems which are not controllable in average but so that each of its truncations is controllable in average. An example of such system is based on the following construction. Let $A \in \mathcal{L}(X)$ and $B \in \mathcal{L}(U, X)$ and assume that the pair $(A, B)$ is controllable, that is to say that $\operatorname{rank}\left(B, A B, \cdots, A^{\operatorname{dim} X-1} B\right)=\operatorname{dim} X$. Let us now consider the probability set $\left(\mathbb{N}^{*}, \mathcal{P}\left(\mathbb{N}^{*}\right), \mu\right)$ with $\mu(\{1\})=\frac{1}{2}$ and for every $\zeta \in \mathbb{N}^{*}, \mu(\{\zeta\})>0$ and define the parameter dependent system:

$$
\dot{y}_{\zeta}=A_{\zeta} y_{\zeta}+B_{\zeta} u \quad\left(\zeta \in \mathbb{N}^{*}\right),
$$

with $A_{\zeta}=A$ for every $\zeta \in \mathbb{N}^{*}, B_{1}=B$ and $B_{\zeta}=-B$ for every $\zeta \in \mathbb{N}^{*} \backslash\{1\}$.

Then, for every $Z \in \mathbb{N}^{*}$, the truncated system is controllable in average. Indeed, we have:

$$
\begin{array}{r}
\operatorname{rank}\left[\sum_{\zeta=1}^{Z}\left(A_{\zeta}\right)^{j} B_{\zeta} \frac{\mu(\{\zeta\})}{\mu(\{1, \cdots, Z\})}, j \in \mathbb{N}\right]=\operatorname{rank}\left[\frac{1}{2} A^{j} B-\left(\sum_{\zeta=2}^{Z} \mu(\{\zeta\})\right) A^{j} B, j \in \mathbb{N}\right] \\
=\operatorname{rank}\left[\mu\left(\mathbb{N}^{*} \backslash\{1, \cdots, Z\}\right) A^{j} B, j \in \mathbb{N}\right]=\operatorname{rank}\left[A^{j} B, j \in \mathbb{N}\right]=\operatorname{dim} X .
\end{array}
$$

But, the full system is not controllable in average. Indeed, for every $\mathrm{z}^{f} \in \mathbb{R}^{n}$ and $T>0$, we have:

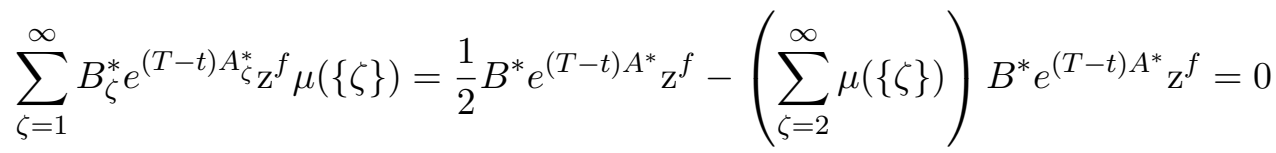

and hence, whatever $T>0$ is, $\int_{0}^{T}\left\|\sum_{\zeta=1}^{\infty} B_{\zeta}^{*} e^{(T-t) A_{\zeta_{\mathrm{Z}}}^{*} \mu(\{\zeta\})}\right\|_{U}^{2} \mathrm{~d} t=0$ does not imply $\mathrm{z}^{f}=0$.

Proof of Proposition 5.1. Set $\theta_{\zeta}=\mu(\{\zeta\})$ without loss of generality, we can assume that $\theta_{\zeta}>0$ for every $\zeta \in \mathbb{N}^{*}$. Set $\theta_{\zeta}^{Z}=\chi_{Z} \mu(\{\zeta\})= \begin{cases}\frac{\theta_{\zeta}}{\sum_{\zeta=1}^{Z} \theta_{\zeta}} & \text { if } \zeta \leqslant Z \\ 0 & \text { otherwise. }\end{cases}$

Let us remind that due to Theorem 3.2, the pairs $\left(A_{\zeta}, B_{\zeta}\right)_{\zeta}$ being controllable in average, is equivalent to $(3.3)$ :

$$
c\left\|\mathrm{z}^{f}\right\|_{X}^{2} \leqslant \int_{0}^{T}\left\|\sum_{\zeta \in \mathbb{N}^{*}} B_{\zeta}^{*} e^{t A_{\zeta}^{*} \mathrm{Z}^{f} \theta_{\zeta}}\right\|_{U}^{2} \mathrm{~d} t \quad\left(\mathrm{z}^{f} \in X\right) .
$$


with $c=c(T)>0$ independent of $\mathrm{z}^{f}$.

But,

$$
\left(\int_{0}^{T}\left\|\sum_{\zeta=1}^{Z} B_{\zeta}^{*} e^{t A_{\zeta}^{*} f^{f}} \theta_{\zeta}^{Z}\right\|_{U}^{2} \mathrm{~d} t\right)^{\frac{1}{2}}=\frac{1}{\sum_{\zeta=1}^{Z} \theta_{\zeta}}\left(\int_{0}^{T}\left\|\sum_{\zeta=1}^{Z} B_{\zeta}^{*} e^{t A_{\zeta_{Z}}^{*} \theta_{\zeta}}\right\|_{U}^{2} \mathrm{~d} t\right)^{\frac{1}{2}}
$$

and using Minkowski inequality,

$$
\begin{aligned}
& \left(\sum_{\zeta=1}^{Z} \theta_{\zeta}\right)\left(\int_{0}^{T}\left\|\sum_{\zeta=1}^{Z} B_{\zeta}^{*} e^{t A_{\zeta_{Z}}^{*} \theta_{\zeta}^{Z}}\right\|_{U}^{2} \mathrm{~d} t\right)^{\frac{1}{2}} \\
& \geqslant\left(\int_{0}^{T}\left\|\sum_{\zeta=1}^{\infty} B_{\zeta}^{*} e^{t A_{\zeta}^{*}{ }^{f} \theta_{\zeta}}\right\|_{U}^{2} \mathrm{~d} t\right)^{\frac{1}{2}}-\left(\int_{0}^{T}\left\|\sum_{\zeta=Z+1}^{\infty} B_{\zeta}^{*} e^{t A_{\zeta_{\mathrm{Z}}}^{*} \theta_{\zeta}}\right\|_{U}^{2} \mathrm{~d} t\right)^{\frac{1}{2}} .
\end{aligned}
$$

From the averaged controllability property, there exists $c>0$ such that:

$$
c\left\|\mathrm{Z}^{f}\right\|_{X}^{2} \leqslant \int_{0}^{T}\left\|\sum_{\zeta=1}^{\infty} B_{\zeta}^{*} e^{t A_{\zeta}^{*} \mathrm{Z}^{f} \theta_{\zeta}}\right\|_{U}^{2} \mathrm{~d} t
$$

and due to the admissibility condition, there exists $C>0$ such that:

$$
\int_{0}^{T}\left\|\sum_{\zeta=Z+1}^{\infty} B_{\zeta}^{*} e^{t A_{\zeta}^{*}} \theta_{\zeta}\right\|_{U}^{2} \mathrm{~d} t \leqslant C\left\|_{\mathrm{Z}}^{f}\right\|_{X}^{2} \sum_{\zeta=Z+1}^{\infty} \theta_{\zeta}
$$

Consequently,

$$
\left(\int_{0}^{T}\left\|\sum_{\zeta=1}^{Z} B_{\zeta}^{*} e^{t A_{\zeta_{\mathrm{Z}}}^{*} \theta_{\zeta}^{Z}}\right\|_{U}^{2} \mathrm{~d} t\right)^{\frac{1}{2}} \geqslant \frac{\sqrt{c}-\sqrt{C\left(1-\sum_{\zeta=1}^{Z} \theta_{\zeta}\right)}}{\sum_{\zeta=1}^{Z} \theta_{\zeta}}\left\|\mathrm{z}^{f}\right\|_{X}
$$

Since $\lim _{Z \rightarrow \infty} \frac{\sqrt{c}-\sqrt{C\left(1-\sum_{\zeta=1}^{Z} \theta_{\zeta}\right)}}{\sum_{\zeta=1}^{Z} \theta_{\zeta}}=\sqrt{c}>0$, we obtain the result.

Proof of Proposition 5.2. As in the previous proof, we set $\theta_{\zeta}=\mu(\{\zeta\})$ and without loss of generality, we can assume that $\theta_{\zeta}>0$ for every $\zeta \in \mathbb{N}^{*}$. Let us then set $\theta_{\zeta}^{Z}=\chi_{Z} \mu(\{\zeta\})= \begin{cases}\frac{\theta_{\zeta}}{\sum_{\zeta=1}^{Z} \theta_{\zeta}} & \text { if } \zeta \leqslant Z, \\ 0 & \text { otherwise. }\end{cases}$

Due to that approximate simultaneous controllability, for every $\varepsilon>0$ and every $\left(\mathrm{y}_{\zeta}^{i}\right)_{\zeta},\left(\mathrm{y}_{\zeta}^{f}\right)_{\zeta} \in$ $L^{2}\left(\mathbb{N}^{*}, X ; \mu\right)$, there exist a control $u \in L^{2}([0, T], U)$ such that:

$$
\sum_{\zeta \in \mathbb{N}^{*}}\left\|y_{\zeta}(T ; u)-\mathrm{y}_{\zeta}^{f}\right\|_{X}^{2} \theta_{\zeta} \leqslant \varepsilon
$$

But we have for every $Z \in \mathbb{N}^{*}$,

$$
\sum_{\zeta \in \mathbb{N}^{*}}\left\|y_{\zeta}(T ; u)-\mathrm{y}_{\zeta}^{f}\right\|_{X}^{2} \theta_{\zeta}^{Z}=\sum_{\zeta=1}^{Z}\left\|y_{\zeta}(T ; u)-\mathrm{y}_{\zeta}^{f}\right\|_{X}^{2} \theta_{\zeta}^{Z} \leqslant \frac{1}{\mu(\{1, \cdots, Z\}} \sum_{\zeta \in \mathbb{N}^{*}}\left\|y_{\zeta}(T ; u)-\mathrm{y}_{\zeta}^{f}\right\|_{X}^{2} \theta_{\zeta} \leqslant \frac{\varepsilon}{\mu(\{1, \cdots, Z\}} .
$$


That is to say that $u \in L^{2}([0, T], U) \rightarrow\left(y_{\zeta}(T ; u)\right)_{\zeta} \in L^{2}\left(\mathbb{N}^{*}, X ; \chi_{Z} \mu\right)$ has a dense image in $L^{2}\left(\mathbb{N}^{*}, X ; \chi_{Z} \mu\right)$. But its image is an affine space and $L^{2}\left(\mathbb{N}^{*}, X ; \chi_{Z} \mu\right)$ is a finite dimensional space. Thus, its image is $L^{2}\left(\mathbb{N}^{*}, X ; \chi_{Z} \mu\right)$ and the truncated system is exactly simultaneously controllable.

Let us finally study the error between the initial minimisation problem:

$$
\begin{array}{cl}
\min & \mathcal{J}_{\kappa}^{\infty}(u):=\frac{1}{2} \int_{0}^{T}\|u(t)\|_{U}^{2} \mathrm{~d} t+\kappa \sum_{\zeta=1}^{\infty}\left\|y_{\zeta}(T ; u)-\mathrm{y}_{\zeta}^{f}\right\|_{X}^{2} \mu(\{\zeta\}) \\
& \sum_{\zeta=1}^{\infty}\left(y_{\zeta}(T ; u)-\mathrm{y}_{\zeta}^{f}\right) \mu(\{\zeta\})=0
\end{array}
$$

and the truncated minimisation problem:

$$
\begin{array}{ll}
\min & \mathcal{J}_{\kappa}^{Z}(u):=\frac{1}{2} \int_{0}^{T}\|u(t)\|_{U}^{2} \mathrm{~d} t+\kappa \sum_{\zeta=1}^{Z}\left\|y_{\zeta}(T ; u)-\mathrm{y}_{\zeta}^{f}\right\|_{X}^{2} \chi_{Z} \mu(\{\zeta\}) \\
& \sum_{\zeta=1}^{Z}\left(y_{\zeta}(T ; u)-\mathrm{y}_{\zeta}^{f}\right) \chi_{Z} \mu(\{\zeta\})=0
\end{array} \quad\left(\kappa \geqslant 0, Z \geqslant Z_{0}\right),
$$

with $Z_{0} \in \mathbb{N}^{*}$ given by Proposition 5.1.

Proposition 5.3. Assume that the system $(1.1)$ is controllable in average for the probability measure $\mu$. Set $\kappa \geqslant 0$. For $Z \geqslant Z_{0}$ (with $Z_{0} \in \mathbb{N}^{*}$ given by Proposition 5.1), let $u_{\kappa}^{Z}$ be the minimizer of the truncated minimisation problem (5.3).

Then, as $Z \rightarrow \infty$, the sequence $\left(u_{\kappa}^{Z}\right)_{Z}$ strongly converges in $L^{2}([0, T], U)$ to the minimizer $u_{\kappa}$ of the initial minimisation problem 5.2.

Proof. Without loss of generality, we can assume that $\mu(\{\zeta\})>0$ for every $\zeta \in \mathbb{N}^{*}$ and for convenience, we set $\mu(\{\zeta\})=\theta_{\zeta}=\theta_{\zeta}^{\infty}$ and as previously, $\theta_{\zeta}^{Z}=\chi_{Z} \mu(\{\zeta\})= \begin{cases}\frac{\theta_{\zeta}}{\sum_{\zeta=1}^{Z} \theta_{\zeta}} & \text { if } \zeta \leqslant Z, \\ 0 & \text { otherwise. }\end{cases}$

Without loss of generality, we can also assume that for every $Z \in \mathbb{N}^{*}$, the system (1.1) in controllable in average for the probability measure $\chi_{Z} \mu$.

Let us introduce for every $Z \in \mathbb{N}^{*} \cup\{\infty\}$ the map $\mathcal{I}^{Z}: L^{2}([0, T], U) \rightarrow\{0, \infty\}$ defined by:

$$
\mathcal{I}^{Z}(u)= \begin{cases}0 & \text { if } \sum_{\zeta=1}^{Z}\left(y_{\zeta}(T ; u)-y_{\zeta}^{f}\right) \theta_{\zeta}^{Z}=0 \\ \infty & \text { otherwise. }\end{cases}
$$

Thus minimizing $\mathcal{J}_{\kappa}^{Z}$ under the constraint $\sum_{\zeta=1}^{Z}\left(y_{\zeta}(T ; u)-\mathrm{y}_{\zeta}^{f}\right) \theta_{\zeta}^{Z}=0$ is equivalent to minimizing $\mathcal{J}_{\kappa}^{Z}+\mathcal{I}^{Z}$.

The proof of this result is based on $\Gamma$-convergence. More precisely, we will prove that the sequence $\left(\mathcal{J}_{\kappa}^{Z}+\mathcal{I}^{Z}\right)_{Z \in \mathbb{N}^{*}} \Gamma$-converge to $\mathcal{J}_{\kappa}^{\infty}+\mathcal{I}^{\infty}$. 


\section{- Upper bound:}

Let $\left(u_{Z}\right)_{Z \in \mathbb{N}^{*}} \in L^{2}([0, T], U)^{\mathbb{N}^{*}}$ be strongly convergent to an element $u_{\infty} \in L^{2}([0, T], U)$.

The aim of this point is to prove:

$$
\mathcal{J}_{\kappa}^{\infty}\left(u_{\infty}\right)+\mathcal{I}^{\infty}\left(u_{\infty}\right) \leqslant \liminf _{Z \rightarrow \infty}\left(\mathcal{J}_{\kappa}^{Z}\left(u_{Z}\right)+\mathcal{I}^{Z}\left(u_{Z}\right)\right)
$$

If $\liminf _{Z \rightarrow \infty} \mathcal{I}^{Z}\left(u_{Z}\right)=\infty$, then, it is clear that (5.4) is true.

Otherwise, we can assume up to the extraction of a subsequence that for every $Z \in \mathbb{N}^{*}$, we have $\mathcal{I}^{Z}\left(u_{Z}\right)=0$. Under this assumption, let us prove:

$$
\mathcal{I}^{\infty}\left(u_{\infty}\right)=0 \quad \text { and } \quad \lim _{Z \rightarrow \infty} \sum_{\zeta=1}^{Z}\left\|y_{\zeta}\left(T ; u_{Z}\right)-\mathrm{y}_{\zeta}^{f}\right\|_{X}^{2} \theta_{\zeta}^{Z}=\sum_{\zeta=1}^{\infty}\left\|y_{\zeta}\left(T ; u_{\infty}\right)-\mathrm{y}_{\zeta}^{f}\right\|_{X}^{2} \theta_{\zeta} .
$$

This will ensure (5.4).

1. Let us prove that $\mathcal{I}^{\infty}\left(u_{\infty}\right)=0$ :

To this end, let us notice:

$$
\begin{aligned}
\sum_{\zeta=1}^{\infty}\left(y_{\zeta}\left(T ; u_{\infty}\right)-\mathrm{y}_{\zeta}^{f}\right) \theta_{\zeta}^{\infty}= & \sum_{\zeta=1}^{\infty}\left(y_{\zeta}\left(T, u_{\infty}\right)-\mathrm{y}_{\zeta}^{f}\right) \theta_{\zeta}^{\infty}-\sum_{\zeta=1}^{\infty}\left(y_{\zeta}\left(T, u_{Z}\right)-\mathrm{y}_{\zeta}^{f}\right) \theta_{\zeta}^{Z} \\
= & \sum_{\zeta=1}^{\infty}\left(y_{\zeta}\left(T, u_{\infty}\right)-\mathrm{y}_{\zeta}^{f}\right) \theta_{\zeta}^{\infty}-\frac{1}{\mu(\{1, \cdots, Z\})} \sum_{\zeta=1}^{\infty}\left(y_{\zeta}\left(T, u_{Z}\right)-\mathrm{y}_{\zeta}^{f}\right) \theta_{\zeta}^{\infty} \\
& +\frac{1}{\mu(\{1, \cdots, Z\})} \sum_{\zeta=Z+1}^{\infty}\left(y_{\zeta}\left(T, u_{Z}\right)-\mathrm{y}_{\zeta}^{f}\right) \theta_{\zeta}^{\infty} \\
= & \sum_{\zeta=1}^{\infty}\left(\int_{0}^{T} e^{\left.(T-t) A_{\zeta} B_{\zeta}\left(u_{\infty}(t)-\frac{u_{Z}(t)}{\mu(\{1, \cdots, Z\})}\right) \mathrm{d} t\right) \theta_{\zeta}^{\infty}}\right. \\
& +\left(1-\frac{1}{\mu(\{1, \cdots, Z\})}\right) \sum_{\zeta=1}^{\infty}\left(e^{\left.T A_{\zeta} \mathrm{y}_{\zeta}^{i}-\mathrm{y}_{\zeta}^{f}\right) \theta_{\zeta}^{\infty}}\right. \\
& +\frac{1}{\mu(\{1, \cdots, Z\})} \sum_{\zeta=Z+1}^{\infty}\left(\int_{0}^{T} e^{(T-t) A_{\zeta}} B_{\zeta} u_{Z}(t) \mathrm{d} t\right) \theta_{\zeta}^{\infty} \\
& +\frac{1}{\mu(\{1, \cdots, Z\})} \sum_{\zeta=Z+1}^{\infty}\left(e^{\left.T A_{\zeta} \mathrm{y}_{\zeta}^{i}-\mathrm{y}_{\zeta}^{f}\right) \theta_{\zeta}^{\infty}}\right.
\end{aligned}
$$

The admissibility condition, ensures:

$$
\begin{aligned}
\left\|\sum_{\zeta=1}^{\infty}\left(\int_{0}^{T} e^{(T-t) A_{\zeta}} B_{\zeta}\left(u_{\infty}(t)-\frac{u_{Z}(t)}{\mu(\{1, \cdots, Z\})}\right) \mathrm{d} t\right) \theta_{\zeta}^{\infty}\right\|_{X}^{2} \\
\leqslant C\left\|u_{\infty}-\frac{u_{Z}}{\mu(\{1, \cdots, Z\})}\right\|_{L^{2}([0, T], U)}^{2},
\end{aligned}
$$


with $C>0$ a constant.

Using Cauchy-Schwarz inequality, we obtain:

$$
\begin{aligned}
\left\|\sum_{\zeta=Z+1}^{\infty}\left(\int_{0}^{T} e^{(T-t) A_{\zeta}} B_{\zeta} u_{Z}(t) \mathrm{d} t\right) \theta_{\zeta}^{\infty}\right\|_{X}^{2} \\
\leqslant(1-\mu(\{1, \cdots, Z\})) \sum_{\zeta=Z+1}^{\infty}\left\|\int_{0}^{T} e^{(T-t) A_{\zeta}} B_{\zeta} u_{Z}(t) \mathrm{d} t\right\|_{X}^{2} \theta_{\zeta}^{\infty} \\
\leqslant(1-\mu(\{1, \cdots, Z\})) \sum_{\zeta=1}^{\infty} \| \int_{0}^{T} e^{(T-t) A_{\zeta} B_{\zeta} u_{Z}(t) \mathrm{d} t \|_{X}^{2} \theta_{\zeta}^{\infty}}
\end{aligned}
$$

But, according to the admissibility conditions (see Lemma 2.2), there exists a constant $\hat{C}>0$ such that:

$$
\left\|\sum_{\zeta=Z+1}^{\infty}\left(\int_{0}^{T} e^{(T-t) A_{\zeta}} B_{\zeta} u_{Z}(t) \mathrm{d} t\right) \theta_{\zeta}^{\infty}\right\|_{X}^{2} \leqslant(1-\mu(\{1, \cdots, Z\})) \hat{C}\left\|u_{Z}\right\|_{L^{2}([0, T], U)}^{2} .
$$

Thus, taking the limit $Z \rightarrow \infty$, we obtain $\left\|\sum_{\zeta=1}^{\infty}\left(y_{\zeta}\left(T ; u_{\infty}\right)-y_{\zeta}^{f}\right) \theta_{\zeta}^{\infty}\right\|_{X}=0$, i.e. $\mathcal{I}^{\infty}\left(u_{\infty}\right)=0$.

2. Let us prove $\lim _{Z \rightarrow \infty} \sum_{\zeta=1}^{Z}\left\|y_{\zeta}\left(T ; u_{Z}\right)-\mathrm{y}_{\zeta}^{f}\right\|_{X}^{2} \theta_{\zeta}^{Z}=\sum_{\zeta=1}^{\infty}\left\|y_{\zeta}\left(T ; u_{\infty}\right)-\mathrm{y}_{\zeta}^{f}\right\|_{X}^{2} \theta_{\zeta}^{\infty}$ :

For every $Z \in \mathbb{N}^{*}$, we have, by Cauchy-Schwarz inequality:

$$
\begin{aligned}
\sum_{\zeta=1}^{Z}\left\|y_{\zeta}\left(T ; u_{Z}\right)-\mathrm{y}_{\zeta}^{f}\right\|_{X}^{2} \theta_{\zeta}^{Z}= & \sum_{\zeta=1}^{Z}\left\|y_{\zeta}\left(T ; u_{Z}\right)-y_{\zeta}\left(T ; u_{\infty}\right)\right\|_{X}^{2} \theta_{\zeta}^{Z}+\sum_{\zeta=1}^{Z}\left\|y_{\zeta}\left(T ; u_{\infty}\right)-\mathrm{y}_{\zeta}^{f}\right\|_{X}^{2} \theta_{\zeta}^{Z} \\
& +2 \sum_{\zeta=1}^{Z}\left\langle y_{\zeta}\left(T ; u_{Z}\right)-y_{\zeta}\left(T ; u_{\infty}\right), y_{\zeta}\left(T ; u_{\infty}\right)-\mathrm{y}_{\zeta}^{f}\right\rangle_{X} \theta_{\zeta}^{Z} \\
\leqslant & \left(\left(\sum_{\zeta=1}^{Z}\left\|y_{\zeta}\left(T ; u_{Z}\right)-y_{\zeta}\left(T ; u_{\infty}\right)\right\|_{X}^{2} \theta_{\zeta}^{Z}\right)^{\frac{1}{2}}\right. \\
& \left.+\left(\sum_{\zeta=1}^{Z}\left\|y_{\zeta}\left(T ; u_{\infty}\right)-\mathrm{y}_{\zeta}^{f}\right\|_{X}^{2} \theta_{\zeta}^{Z}\right)^{\frac{1}{2}}\right)^{2}
\end{aligned}
$$

Using the admissibility of every system indexed by $\zeta$, for every $\zeta \in \mathbb{N}^{*}$, there exists $C_{\zeta}>0$ such that:

$$
\sum_{\zeta=1}^{Z}\left\|y_{\zeta}\left(T ; u_{Z}\right)-y_{\zeta}\left(T ; u_{\infty}\right)\right\|_{X}^{2} \theta_{\zeta}^{Z} \leqslant \sum_{\zeta=1}^{Z} C_{\zeta} \theta_{\zeta}^{Z}\left\|u_{Z}-u_{\infty}\right\|_{L^{2}([0, T], U)}^{2}
$$


In addition, due to assumption (2.3) made in Lemma 2.2, we have $\lim _{Z \rightarrow \infty} \sum_{\zeta=1}^{Z} C_{\zeta} \theta_{\zeta}^{Z}<\infty$ and hence, since $\left(u_{Z}\right)_{Z}$ is strongly convergent to $u_{\infty}$

$$
\lim _{Z \rightarrow \infty} \sum_{\zeta=1}^{Z}\left\|y_{\zeta}\left(T ; u_{Z}\right)-y_{\zeta}\left(T ; u_{\infty}\right)\right\|_{X}^{2} \theta_{\zeta}^{Z}=0
$$

On the other hand, it remains clear, due to the construction of $\theta_{\zeta}^{Z}$ that:

$$
\lim _{Z \rightarrow \infty} \sum_{\zeta=1}^{Z}\left\|y_{\zeta}\left(T ; u_{\infty}\right)-\mathrm{y}_{\zeta}^{f}\right\|_{X}^{2} \theta_{\zeta}^{Z}=\sum_{\zeta=1}^{\infty}\left\|y_{\zeta}\left(T ; u_{\infty}\right)-\mathrm{y}_{\zeta}^{f}\right\|_{X}^{2} \theta_{\zeta}^{\infty}
$$

Thus,

$$
\lim _{Z \rightarrow \infty} \sum_{\zeta=1}^{Z}\left\|y_{\zeta}\left(T ; u_{Z}\right)-\mathrm{y}_{\zeta}^{f}\right\|_{X}^{2} \theta_{\zeta}^{Z}=\sum_{\zeta=1}^{\infty}\left\|y_{\zeta}\left(T ; u_{\infty}\right)-\mathrm{y}_{\zeta}^{f}\right\|_{X}^{2} \theta_{\zeta}^{\infty}
$$

\section{- Lower bound:}

Set $u_{\infty} \in L^{2}([0, T], U)$. The aim is to prove that there exists a sequence $\left(u_{Z}\right)_{Z \in \mathbb{N}^{*}}$ strongly convergent to $u_{\infty}$ such that:

$$
\mathcal{J}_{\kappa}^{\infty}\left(u_{\infty}\right)+\mathcal{I}^{\infty}\left(u_{\infty}\right) \geqslant \limsup _{Z \rightarrow \infty}\left(\mathcal{J}_{\kappa}^{Z}\left(u_{\infty}\right)+\mathcal{I}^{Z}\left(u_{\infty}\right)\right)
$$

If $\mathcal{I}^{\infty}\left(u_{\infty}\right)=\infty$ then this result can be easily obtained with $u_{Z}=u_{\infty}$.

Let us now assume that $\mathcal{I}^{\infty}\left(u_{\infty}\right)=0$. From the previous point, it remains clear that if the sequence $\left(u_{Z}\right)_{Z}$ is converging to $u_{\infty}$ and if for every $Z \in \mathbb{N}^{*}, \mathcal{I}^{Z}\left(u_{Z}\right)=0$ then:

$$
\mathcal{J}_{\kappa}^{\infty}\left(u_{\infty}\right)=\lim _{Z \rightarrow \infty} \mathcal{J}_{\kappa}^{Z}\left(u_{Z}\right) .
$$

Thus we only need to prove that such a sequence $\left(u_{Z}\right)_{Z}$ exists.

Let us write $u_{Z}=u_{\infty}+v_{Z}$. Then $\mathcal{I}^{Z}\left(u_{Z}\right)=0$ means:

$$
\sum_{\zeta=1}^{Z} \int_{0}^{T} e^{(T-t) A_{\zeta}} B_{\zeta} v_{Z}(t) \mathrm{d} t \theta_{\zeta}^{Z}=-\sum_{\zeta=1}^{Z}\left(y_{\zeta}\left(T ; u_{\infty}\right)-\mathrm{y}_{\zeta}^{f}\right) \theta_{\zeta}^{Z}
$$

Since we have assumed that the system 1.1 is controllable in average, such a $v_{Z}$ exists and in addition, there exists a constant $C>0$ independent of $v_{Z}$ such that:

$$
\left\|v_{Z}\right\|_{L^{2}([0, T], U)}^{2} \leqslant C\left\|\sum_{\zeta=1}^{Z}\left(y_{\zeta}\left(T ; u_{\infty}\right)-\mathrm{y}_{\zeta}^{f}\right) \theta_{\zeta}^{Z}\right\|_{X}^{2} .
$$


But since $\sum_{\zeta \in \mathbb{N}^{*}}\left(y_{\zeta}\left(T ; u_{\infty}\right)-\mathrm{y}_{\zeta}^{f}\right) \theta_{\zeta}^{\infty}=0$, we have:

$$
\begin{array}{r}
\left\|\sum_{\zeta=1}^{Z}\left(y_{\zeta}\left(T ; u_{\infty}\right)-\mathrm{y}_{\zeta}^{f}\right) \theta_{\zeta}^{Z}\right\|_{X}=\left\|\sum_{\zeta=1}^{\infty}\left(y_{\zeta}\left(T ; u_{\infty}\right)-\mathrm{y}_{\zeta}^{f}\right)\left(\theta_{\zeta}^{\infty}-\theta_{\zeta}^{Z}\right)\right\|_{X} \\
\quad \leqslant \frac{1-\mu(\{1, \cdots, Z\})}{\mu(\{1, \cdots, Z\})}\left\|\sum_{\zeta=1}^{Z}\left(y_{\zeta}\left(T ; u_{\infty}\right)-\mathrm{y}_{\zeta}^{f}\right) \theta_{\zeta}^{\infty}\right\|_{X}+\left\|\sum_{\zeta=Z+1}^{\infty}\left(y_{\zeta}\left(T ; u_{\infty}\right)-\mathrm{y}_{\zeta}^{f}\right) \theta_{\zeta}^{\infty}\right\|_{X},
\end{array}
$$

which is going to 0 when $Z \rightarrow \infty$. Consequently, $\left(v_{Z}\right)_{Z}$ converges to 0 , that is to say, there exists a sequence $\left(u_{Z}\right)_{Z}$ convergent to $u_{\infty}$ such that $\mathcal{I}^{Z}\left(u_{Z}\right)=0$ for every $Z \geqslant 1$.

The final result follows from $\Gamma$-convergence property and $\mathcal{J}_{\kappa}^{\infty}+\mathcal{I}^{\infty}$ admits one and only one minimizer.

Let us denote by $u_{\kappa}^{Z}$ (resp. $u_{\kappa}^{\infty}$ ) the minimizer of the truncated (resp. initial) minimisation problem. We proved here that $\lim _{Z \rightarrow \infty} u_{\kappa}^{Z}=u_{\kappa}^{\infty}$. Thus, if $\lim _{\kappa \rightarrow \infty} u_{\kappa}^{\infty}=u_{\infty}^{\infty}$ exists, we have: $\lim _{\kappa \rightarrow \infty} \lim _{Z \rightarrow \infty} u_{\kappa}^{Z}=u_{\infty}^{\infty}$. But, do we have $\lim _{Z \rightarrow \infty} \lim _{\kappa \rightarrow \infty} u_{\kappa}^{Z}=u_{\infty}^{\infty}$ ? This question is the aim of the next proposition.

Proposition 5.4. Let us assume that the system (1.1) is controllable in average for the probability measure $\mu$.

Then,

1. For every $\kappa \geqslant 0$ and every large enough $Z \in \mathbb{N}^{*}$, there exists a minimizer $u_{\kappa}^{Z} \in L^{2}([0, T], U)$ of the truncated minimisation problem (5.3).

2. Up to a subsequence, the sequence $\left(u_{\kappa}^{Z}\right)_{\kappa}$ is strongly convergent to an element $u_{\infty}^{Z} \in L^{2}([0, T], U)$ which is a solution of the minimisation problem:

$$
\begin{array}{cc}
\min & \frac{1}{2}\|u\|_{L^{2}([0, T], U)}^{2} \\
& y_{\zeta}(T)=\mathrm{y}_{\zeta}^{Z, \star}
\end{array} \quad\left(\zeta \in \mathbb{N}^{*} \quad \chi_{Z} \mu-\text { a.e. }\right),
$$

where $\mathrm{y}_{\zeta}^{Z, \star}$ is a minimizer of the minimization problem:

$$
\begin{array}{cl}
\min & \left\|\mathrm{y}_{\zeta}-\mathrm{y}_{\zeta}^{f}\right\|_{L^{2}\left(\Omega, X ; \chi_{z} \mu\right)} \\
& \left(\mathrm{y}_{\zeta}\right)_{\zeta} \in \overline{\left\{y_{\zeta}(T ; u), u \in L^{2}([0, T], U)\right\}} \\
& \mathbb{E}^{Z}\left(\mathrm{y}_{\zeta}\right)_{\zeta}=\mathbb{E}^{Z}\left(\mathrm{y}_{\zeta}^{f}\right)_{\zeta}
\end{array}
$$

and where we have set:

$$
\mathbb{E}^{Z}\left(\mathrm{y}_{\zeta}\right)=\sum_{\zeta \in \mathbb{N}^{*}} \mathrm{y}_{\zeta} \chi_{Z} \mu(\{\zeta\}) \quad\left(\left(\mathrm{y}_{\zeta}\right)_{\zeta} \in L^{2}\left(\mathbb{N}^{*}, X ; \chi_{Z} \mu\right)\right)
$$

3. Moreover,

(a) $\lim _{Z \rightarrow \infty} \mathrm{y}_{\zeta}^{Z, \star}=\mathrm{y}_{\zeta}^{\star}$, with $\mathrm{y}_{\zeta}^{\star} \in L^{2}(\Omega, X ; \mu)$ given by Theorem 4.1. 
(b) if the sequence $\left(u_{\infty}^{Z}\right)_{Z}$ is bounded, then the system (1.1) can be exactly steered from $\mathrm{y}_{\zeta}^{i}$ to $\mathrm{y}_{\zeta}^{\star}$ and up to a subsequence $\left(u_{\infty}^{Z}\right)_{Z}$ it is weakly convergent to such a control; otherwise, the system (1.1) can be approximatively steered from $\mathrm{y}_{\zeta}^{i}$ to $\mathrm{y}_{\zeta}^{\star}$.

Proof. First of all, the existence of $u_{\kappa}^{Z}$ for every $\kappa \geqslant 0$ and every $Z$ large enough is ensured by Proposition 5.1.

Without loss of generality, we can assume $\mu(\{\zeta\})>0$ for every $\zeta \in \mathbb{N}^{*}$ and the system (1.1) is controllable in average for the measure $\chi_{Z} \mu$ for every $Z \in \mathbb{N}^{*}$. As in the previous proofs, we set for convenience, $\mu(\{\zeta\})=\theta_{\zeta}=\theta_{\zeta}^{\infty}$ and $\theta_{\zeta}^{Z}=\chi_{Z} \mu(\{\zeta\})= \begin{cases}\frac{\theta_{\zeta}}{\sum_{\zeta=1}^{Z} \theta_{\zeta}} & \text { if } \zeta \leqslant Z, \\ 0 & \text { otherwise. }\end{cases}$

Finally, changing $\mathrm{y}_{\zeta}^{f}$ in $\mathrm{y}_{\zeta}^{f}-e^{T A_{\zeta}} \mathrm{y}_{\zeta}^{i}$, we can assume without loss of generality that $\mathrm{y}_{\zeta}^{i}=0$.

Let us notice that for every $Z \geqslant 1$, the control system (1.1) endowed with the measure $\chi_{z} \mu$ can be recast as a parameter dependent system whose parameters take place in a set of finite cardinal. Consequently, Corollary 4.1 ensure that the sequence of minimizers $\left(u_{\kappa}^{Z}\right)_{\kappa \geqslant 0}$ is convergent to $u_{\infty}^{Z} \in L^{2}([0, T], U)$ solution of the minimisation problem (5.5).

Let us prove item $3 \mathrm{a}$.

For every $Z \in \mathbb{N}^{*} \cup \infty$, the minimisations problems $(5.6)$ is:

$$
\begin{array}{ll}
\min & \mathcal{G}^{Z}\left(\mathrm{y}_{\zeta}\right)=\sum_{\zeta=1}^{Z}\left\|\mathrm{y}_{\zeta}-\mathrm{y}_{\zeta}^{f}\right\|_{X}^{2} \theta_{\zeta}^{Z} \\
& \mathrm{y}_{\zeta} \in \overline{\left\{\left(y_{\zeta}(T ; u)\right)_{\zeta}, u \in L^{2}([0, T], U)\right\}} \\
& \sum_{\zeta=1}^{Z}\left(\mathrm{y}_{\zeta}-\mathrm{y}_{\zeta}^{f}\right) \theta_{\zeta}^{Z}=0 .
\end{array}
$$

Define

$$
\begin{aligned}
& \mathcal{I}_{0}\left(\mathrm{y}_{\zeta}\right)= \begin{cases}0 & \text { if } \mathrm{y}_{\zeta} \in \overline{\left\{\left(y_{\zeta}(T ; u)\right)_{\zeta}, u \in L^{2}([0, T], U)\right\}} \\
\infty & \text { otherwise }\end{cases} \\
& \text { and } \quad \mathcal{I}_{1}^{Z}\left(\mathrm{y}_{\zeta}\right)= \begin{cases}0 & \text { if } \sum_{\zeta=1}^{Z}\left(\mathrm{y}_{\zeta}-\mathrm{y}_{\zeta}^{f}\right) \theta_{\zeta}^{Z}=0, \\
\infty & \text { otherwise }\end{cases}
\end{aligned}
$$

so that the above minimisation problem is:

$$
\min _{\mathrm{y}_{\zeta} \in L^{2}\left(\mathbb{N}^{*}, X ; \mu\right)}\left(\mathcal{G}^{Z}\left(\mathrm{y}_{\zeta}\right)+\mathcal{I}_{0}\left(\mathrm{y}_{\zeta}\right)+\mathcal{I}_{1}^{Z}\left(\mathrm{y}_{\zeta}\right)\right) .
$$

In the next points, we will prove that $\left(\mathcal{G}^{Z}+\mathcal{I}_{0}+\mathcal{I}_{1}^{Z}\right)_{Z} \Gamma$-convergence to $\mathcal{G}^{\infty}+\mathcal{I}_{0}+\mathcal{I}_{1}^{\infty}$.

- Lower bound:

Let $\left(\left(\mathrm{y}_{\zeta}^{Z}\right)_{\zeta}\right)_{Z} \in L^{2}\left(\mathbb{N}^{*}, X ; \mu\right)^{N^{*}}$ be a convergent sequence in $L^{2}\left(\mathbb{N}^{*}, X ; \mu\right)$ to $\left(\mathrm{y}_{\zeta}^{\infty}\right)_{\zeta}$. The aim is to prove:

$$
\mathcal{G}^{Z}\left(\mathrm{y}_{\zeta}^{\infty}\right)+\mathcal{I}_{0}\left(\mathrm{y}_{\zeta}^{\infty}\right)+\mathcal{I}_{1}^{\infty}\left(\mathrm{y}_{\zeta}^{\infty}\right) \leqslant \liminf _{Z \rightarrow \infty} \mathcal{G}^{Z}\left(\mathrm{y}_{\zeta}^{Z}\right)+\mathcal{I}_{0}\left(\mathrm{y}_{\zeta}^{Z}\right)+\mathcal{I}_{1}^{Z}\left(\mathrm{y}_{\zeta}^{Z}\right)
$$


First of all, if $\liminf _{Z \rightarrow \infty} \mathcal{G}^{Z}\left(\mathrm{y}_{\zeta}^{Z}\right)+\mathcal{I}_{0}\left(\mathrm{y}_{\zeta}^{Z}\right)+\mathcal{I}_{1}^{Z}\left(\mathrm{y}_{\zeta}^{Z}\right)=\infty$, the result is obvious. Consequently, we can assume $\mathcal{I}_{0}\left(\mathrm{y}_{\zeta}^{Z}\right)+\mathcal{I}_{1}^{Z}\left(\mathrm{y}_{\zeta}^{Z}\right)=0$ for every $Z \in \mathbb{N}^{*}$.

Since $\mathcal{I}_{0}\left(\mathrm{y}_{\zeta}^{Z}\right)=0$ for every $Z \in \mathbb{N}^{*}$ and since $\overline{\left\{\left(y_{\zeta}(T ; u)\right)_{\zeta}, u \in L^{2}([0, T], U)\right\}}$ is a closed set, then $\mathcal{I}_{0}\left(\mathrm{y}_{\zeta}^{\infty}\right)=0$.

Let us now prove that $\mathcal{I}_{1}^{\infty}\left(\mathrm{y}_{\zeta}^{\infty}\right)=0$. To this end, we notice that:

$$
\begin{aligned}
\left\|\sum_{\zeta=1}^{\infty}\left(\mathrm{y}_{\zeta}^{\infty}-\mathrm{y}_{\zeta}^{f}\right) \theta_{\zeta}^{\infty}\right\|_{X} & =\left\|\sum_{\zeta=1}^{\infty}\left(\mathrm{y}_{\zeta}^{\infty}-\mathrm{y}_{\zeta}^{Z}\right) \theta_{\zeta}^{\infty}+\left(\sum_{\zeta=1}^{Z} \theta_{\zeta}^{\infty}\right)\left(\sum_{\zeta=1}^{Z}\left(\mathrm{y}_{\zeta}^{Z}-\mathrm{y}_{\zeta}^{f}\right) \theta_{\zeta}^{Z}\right)+\sum_{\zeta=Z+1}^{\infty}\left(\mathrm{y}_{\zeta}^{Z}-\mathrm{y}_{\zeta}^{f}\right) \theta_{\zeta}^{\infty}\right\|_{X} \\
& =\left\|\sum_{\zeta=1}^{\infty}\left(\mathrm{y}_{\zeta}^{\infty}-\mathrm{y}_{\zeta}^{Z}\right) \theta_{\zeta}^{\infty}+\sum_{\zeta=Z+1}^{\infty}\left(\mathrm{y}_{\zeta}^{Z}-\mathrm{y}_{\zeta}^{f}\right) \theta_{\zeta}^{\infty}\right\|_{X} \\
& \leqslant\left\|\sum_{\zeta=1}^{\infty}\left(\mathrm{y}_{\zeta}^{\infty}-\mathrm{y}_{\zeta}^{Z}\right) \theta_{\zeta}^{\infty}\right\|_{X}+\left\|\sum_{\zeta=Z+1}^{\infty}\left(\mathrm{y}_{\zeta}^{Z}-\mathrm{y}_{\zeta}^{f}\right) \theta_{\zeta}^{\infty}\right\|_{X} .
\end{aligned}
$$

Thus, taking the limit $Z \rightarrow \infty$, we obtain $\sum_{\zeta=1}^{\infty}\left(\mathrm{y}_{\zeta}^{\infty}-\mathrm{y}_{\zeta}^{f}\right) \theta_{\zeta}^{\infty}=0$, i.e. $\mathcal{I}_{1}^{\infty}\left(\mathrm{y}_{\zeta}^{\infty}\right)=0$.

To conclude, it is obvious that $\lim _{Z \rightarrow \infty} \mathcal{G}^{Z}\left(\mathrm{y}_{\zeta}^{Z}\right)=\mathcal{G}^{\infty}\left(\mathrm{y}_{\zeta}^{\infty}\right)$.

\section{- Upper bound:}

Let $\left(\mathrm{y}_{\zeta}^{\infty}\right)_{\zeta} \in L^{2}\left(\mathbb{N}^{*}, X ; \mu\right)$, the aim is to prove that there exists $\left(\left(\mathrm{y}_{\zeta}^{Z}\right)_{\zeta}\right)_{Z} \in L^{2}\left(\mathbb{N}^{*}, X ; \mu\right)^{N^{*}}$, a sequence converging to $\left(\mathrm{y}_{\zeta}^{\infty}\right)_{\zeta}$ such that:

$$
\mathcal{G}^{Z}\left(\mathrm{y}_{\zeta}^{\infty}\right)+\mathcal{I}_{0}\left(\mathrm{y}_{\zeta}^{\infty}\right)+\mathcal{I}_{1}^{\infty}\left(\mathrm{y}_{\zeta}^{\infty}\right) \geqslant \limsup _{Z \rightarrow \infty} \mathcal{G}^{Z}\left(\mathrm{y}_{\zeta}^{Z}\right)+\mathcal{I}_{0}\left(\mathrm{y}_{\zeta}^{Z}\right)+\mathcal{I}_{1}^{Z}\left(\mathrm{y}_{\zeta}^{Z}\right)
$$

If $\mathcal{I}_{0}\left(\mathrm{y}_{\zeta}^{\infty}\right)=\infty$, the result is clear with $\mathrm{y}_{\zeta}^{Z}=\mathrm{y}_{\zeta}^{\infty}$.

If $\mathcal{I}_{1}^{\infty}\left(\mathrm{y}_{\zeta}^{\infty}\right)=\infty$, i.e. there exists $\varepsilon>0$ such that $\left\|\sum_{\zeta=1}^{\infty}\left(\mathrm{y}_{\zeta}^{\infty}-\mathrm{y}_{\zeta}^{f}\right) \theta_{\zeta}^{\infty}\right\|_{X} \geqslant \varepsilon$. Consider the sequence $\left(\left(\mathrm{y}_{\zeta}^{Z}\right)_{\zeta}\right)_{Z}$ given by $\mathrm{y}_{\zeta}^{Z}=\left\{\begin{array}{ll}\mathrm{y}_{\zeta}^{\infty} & \text { if } \zeta \leqslant Z, \\ 0 & \text { otherwise. }\end{array}\right.$ Then $\left(\left(\mathrm{y}_{\zeta}^{Z}\right)_{\zeta}\right)_{Z}$ converges to $\left(\mathrm{y}_{\zeta}^{\infty}\right)_{\zeta}$ in $L^{2}\left(\mathbb{N}^{*}, X ; \mu\right)$ as $Z \rightarrow \infty$ and

$$
\begin{aligned}
\left\|\sum_{\zeta=1}^{Z}\left(\mathrm{y}_{\zeta}^{Z}-\mathrm{y}_{\zeta}^{f}\right) \theta_{\zeta}^{Z}\right\|_{X} & =\frac{1}{\sum_{\zeta=1}^{Z} \theta_{\zeta}^{\infty}}\left\|\sum_{\zeta=1}^{\infty}\left(\mathrm{y}_{\zeta}^{Z}-\mathrm{y}_{\zeta}^{\infty}+\mathrm{y}_{\zeta}^{\infty}-\mathrm{y}_{\zeta}^{f}\right) \theta_{\zeta}^{\infty}\right\|_{X} \\
& \geqslant \frac{1}{\sum_{\zeta=1}^{Z} \theta_{\zeta}^{\infty}}\left(\left\|\sum_{\zeta=1}^{\infty}\left(\mathrm{y}_{\zeta}^{\infty}-\mathrm{y}_{\zeta}^{f}\right) \theta_{\zeta}^{\infty}\right\|_{X}-\left\|\sum_{\zeta=1}^{\infty}\left(\mathrm{y}_{\zeta}^{\infty}-\mathrm{y}_{\zeta}^{Z}\right) \theta_{\zeta}^{\infty}\right\|_{X}\right) \\
& \geqslant \frac{1}{\sum_{\zeta=1}^{Z} \theta_{\zeta}^{\infty}}\left(\varepsilon-\sqrt{\sum_{\zeta=1}^{\infty}\left\|\mathrm{y}_{\zeta}^{\infty}-\mathrm{y}_{\zeta}^{Z}\right\|_{X}^{2} \theta_{\zeta}^{\infty}}\right) .
\end{aligned}
$$


But, since $\left(\left(\mathrm{y}_{\zeta}^{Z}\right)_{\zeta}\right)_{Z}$ converges to $\left(\mathrm{y}_{\zeta}^{\infty}\right)_{\zeta}$, we have for $Z$ large enough, $\left\|\sum_{\zeta=1}^{Z}\left(\mathrm{y}_{\zeta}^{Z}-\mathrm{y}_{\zeta}^{f}\right) \theta_{\zeta}^{Z}\right\|_{X} \geqslant \frac{\varepsilon}{2}$, that is to say $\mathcal{I}_{1}^{Z}\left(\mathrm{y}_{\zeta}^{Z}\right)=\infty$.

Now assume that $\mathcal{I}_{0}\left(\mathrm{y}_{\zeta}^{\infty}\right)=\mathcal{I}_{1}^{\infty}\left(\mathrm{y}_{\zeta}^{\infty}\right)=0$. First of all, it is easy to show that if the sequence $\left(\left(\mathrm{y}_{\zeta}^{Z}\right)_{\zeta}\right)_{Z}$ converges to $\left(\mathrm{y}_{\zeta}^{\infty}\right)_{\zeta}$ then $\lim _{Z \rightarrow \infty} \mathcal{G}^{Z}\left(\mathrm{y}_{\zeta}^{Z}\right)=\mathcal{G}^{\infty}\left(\mathrm{y}_{\zeta}^{\infty}\right)$. Consequently, in order to prove (5.7), we only need to prove the existence of a sequence $\left(\left(\mathrm{y}_{\zeta}^{Z}\right)_{\zeta}\right)_{Z} \in L^{2}\left(\mathbb{N}^{*}, X ; \mu\right)^{\mathbb{N}^{*}}$ convergent to $\left(\mathrm{y}_{\zeta}^{\infty}\right)_{\zeta}$ such that $\mathcal{I}_{0}\left(\mathrm{y}_{\zeta}^{Z}\right)=\mathcal{I}_{1}^{Z}\left(\mathrm{y}_{\zeta}^{Z}\right)=0$ for every large enough $Z$.

Since $\mathrm{y}_{\zeta}^{\infty} \in \overline{\left\{\left(y_{\zeta}(T ; u)\right)_{\zeta}, u \in L^{2}([0, T], U)\right\}}$, there exists a sequence $\left(u_{Z}\right)_{Z} \in L^{2}([0, T], U)^{\mathbb{N}^{*}}$ such that $\lim _{Z \rightarrow \infty} \sum_{\zeta=1}^{\infty}\left\|y_{\zeta}\left(T ; u_{Z}\right)-\mathrm{y}_{\zeta}^{\infty}\right\| \theta_{\zeta}^{\infty}=0$ and in addition, since $\mathbb{E}_{\zeta}^{\infty}=\mathbb{E}_{\zeta}^{f}$, we have $\lim _{Z \rightarrow \infty} \sum_{\zeta=1}^{\infty}\left(y_{\zeta}\left(T ; u_{Z}\right)-\right.$ $\left.\mathrm{y}_{\zeta}^{f}\right) \theta_{\zeta}^{\infty}=0$. Moreover, the system (1.1) is controllable in average for the measure $\chi_{Z} \mu$, thus the minimisation problem:

$$
\begin{array}{cc}
\min & \frac{1}{2}\|v\|_{L^{2}([0, T], U)}^{2} \\
& \mathbb{E}^{Z}\left(y_{\zeta}(T ; v)\right)_{\zeta}=\mathbb{E}^{Z}\left(\mathrm{y}_{\zeta}^{f}-y_{\zeta}\left(T ; u_{Z}\right)\right)_{\zeta},
\end{array}
$$

admits a minimum which is obtained for $v=v_{Z}$. In addition, since $\lim _{Z \rightarrow \infty} \mathbb{E}^{Z}\left(\mathrm{y}_{\zeta}^{f}-y_{\zeta}\left(T ; u_{Z}\right)\right)_{\zeta}=0$, we obtain $\lim _{Z \rightarrow \infty}\left\|v_{Z}\right\|_{L^{2}([0, T], U)}=0$. Consequently, we have build a sequence $\left(\left(y_{\zeta}\left(T ; u_{Z}+v_{Z}\right)\right)_{\zeta}\right)_{Z}$, satisfying $\mathcal{I}_{0}\left(y_{\zeta}\left(T ; u_{Z}+v_{Z}\right)\right)=\mathcal{I}_{1}^{Z}\left(y_{\zeta}\left(T ; u_{Z}+v_{Z}\right)\right)=0$ for every $Z \in \mathbb{N}^{*}$ and convergent to $\left(\mathrm{y}_{\zeta}^{\infty}\right)_{\zeta}$, since,

$$
\left\|\mathrm{y}_{\zeta}^{\infty}-y_{\zeta}\left(T ; u_{Z}+v_{Z}\right)\right\|_{L^{2}\left(\mathbb{N}^{*}, X ; \mu\right)} \leqslant\left\|\mathrm{y}_{\zeta}^{\infty}-y_{\zeta}\left(T ; u_{Z}\right)\right\|_{L^{2}\left(\mathbb{N}^{*}, X ; \mu\right)}+\left\|y_{\zeta}\left(T ; v_{Z}\right)\right\|_{L^{2}\left(\mathbb{N}^{*}, X ; \mu\right)}
$$

is going to 0 as $Z \rightarrow \infty$.

All in all, from $\Gamma$-convergence tools and the fact that $\mathcal{G}^{\infty}+\mathcal{I}_{0}+\mathcal{I}_{1}^{\infty}$ admits one and only one minimizer, we obtain $\lim _{Z \rightarrow \infty}\left\|\mathrm{y}_{\zeta}^{Z, \star}-\mathrm{y}_{\zeta}^{\star}\right\|_{L^{2}\left(\mathbb{N}^{*}, X ; \mu\right)}=0$.

Let us finally prove item $3 \mathrm{~b}$.

Firstly, we have for every $Z \in \mathbb{N}^{*}, y_{\zeta}\left(T ; u_{\infty}^{Z}\right)=\mathrm{y}_{\zeta}^{Z, \star}$ and hence, from the above point, the sequence $\left(\left(y_{\zeta}\left(T ; u_{\infty}^{Z}\right)\right)_{\zeta}\right)_{Z}$ is strongly convergent to $\left(\mathrm{y}_{\zeta}^{\star}\right)_{\zeta}$ in $L^{2}\left(\mathbb{N}^{*}, X ; \mu\right)$.

In addition, if the sequence $\left(u_{\infty}^{Z}\right)_{Z}$ is bounded, then up to a subsequence, this sequence is weakly convergent to a control $u_{\infty}^{\infty}$ and hence the sequence $\left(\left(y_{\zeta}\left(T ; u_{\infty}^{Z}\right)\right)_{\zeta}\right)_{Z}$ is weakly convergent to $\left(y_{\zeta}\left(T ; u_{\infty}^{\infty}\right)\right)_{\zeta}$ in $L^{2}\left(\mathbb{N}^{*}, X ; \mu\right)$. But from the above point, the sequence $\left(\left(\mathrm{y}_{\zeta}^{Z, \star}\right)_{\zeta}\right)_{Z}=\left(\left(y_{\zeta}\left(T ; u_{\infty}^{Z}\right)\right)_{\zeta}\right)_{Z}$ is convergent to $\left(\mathrm{y}_{\zeta}^{\star}\right)_{\zeta}$. Thus, $y_{\zeta}\left(T ; u_{\infty}^{\infty}\right)=\mathrm{y}_{\zeta}^{\star}$.

\section{Concluding remarks}

In this paper, we have presented a theoretical link between the averaged controllability and the exact simultaneous controllability. But there still exist many practical questions to be addressed. We list here some of them: 
- The problem of convergence rates both for variances and controls as $\kappa \rightarrow \infty$ is open. Such results would be helpful in order to validate numerical simulations, since from a computational viewpoint, it is hard to determine what the decay or convergence rate is or even if the limit vanishes or not.

- When the probability space $\Omega$ is of infinite cardinal, we have introduced a truncation parameter $Z$. In that case, we have to parameters $(Z$ and $\kappa)$ going to infinity. Propositions 5.3 and 5.4 show that the limits in $\kappa$ and $Z$ commute. But, in practice, it would be interesting to be in condition to bound the analysis and simulations to deal with a single parameter. To this end, we should establish some explicit relation between both of them, for instance, find a function $Z \mapsto \kappa(Z)$ such that when letting $Z \rightarrow \infty$, the correct asymptotic behavior in ensured. This problem is related to the one of convergence rates mentioned in the previous item.

- Similar results as those in section 5 could be obtained with a continuous measure and under Lipschitz-regularity assumptions on $\zeta \mapsto\left(A_{\zeta}, B_{\zeta}\right)$. In this situation, instead of truncating the system, one could use the approximation of Lipschitz functions by piecewise constant functions.

- Finally, the penalization procedure proposed here could be extended in the PDE context.

\section{References}

[1] Boyer, Franck. On the penalised hum approach and its applications to the numerical approximation of null-controls for parabolic problems. ESAIM: Proc., 41:15-58, 2013.

[2] L. A. Fernández and E. Zuazua. Approximate controllability for the semilinear heat equation involving gradient terms. J. Optim. Theory Appl., 101(2):307-328, 1999.

[3] U. Helmke and M. Schönlein. Uniform ensemble controllability for one-parameter families of timeinvariant linear systems. Syst. Control Lett., 71:69-77, 2014.

[4] M. Lazar and E. Zuazua. Averaged control and observation of parameter-depending wave equations. C. R. Math. Acad. Sci. Paris, 352(6):497-502, 2014.

[5] J.-S. Li. Ensemble control of finite-dimensional time-varying linear systems. IEEE Trans. Automat. Control, 56(2):345-357, 2011.

[6] J.-S. Li and N. Khaneja. Ensemble control of Bloch equations. IEEE Trans. Automat. Control, 54(3):528-536, 2009.

[7] J.-L. Lions. Contrôlabilité exacte, perturbations et stabilisation de systèmes distribués. Tome 1, volume 8 of Recherches en Mathématiques Appliquées [Research in Applied Mathematics]. Masson, Paris, 1988. Contrôlabilité exacte. [Exact controllability], With appendices by E. Zuazua, C. Bardos, G. Lebeau and J. Rauch.

[8] J.-L. Lions. Remarques sur la controlâbilite approchée. In Spanish-French Conference on DistributedSystems Control (Spanish) (Málaga, 1990), pages 77-87. Univ. Málaga, Málaga, 1990.

[9] J. Lohéac and E. Zuazua. Averaged controllability of parameter dependent wave equations. submitted, Mar. 2015. 
[10] I. R. Petersen. A notion of possible controllability for uncertain linear systems with structured uncertainty. Automatica J. IFAC, 45(1):134-141, 2009.

[11] D. L. Russell. The Dirichlet-Neumann boundary control problem associated with Maxwell's equations in a cylindrical region. SIAM J. Control Optim., 24(2):199-229, 1986.

[12] A. V. Savkin and I. R. Petersen. Uncertainty-averaging approach to output feedback optimal guaranteed cost control of uncertain systems. J. Optim. Theory Appl., 88(2):321-337, 1996.

[13] S. G. Schirmer, I. C. H. Pullen, and A. I. Solomon. Controllability of multi-partite quantum systems and selective excitation of quantum dots. Journal of Optics B: Quantum and Semiclassical Optics, 7(10):S293, 2005.

[14] L. Schwartz. Étude des sommes d'exponentielles réelles. Actualités scientifiques et industrielles. 959. Paris: Hermann \& Cie. 89 p. (1943)., 1943.

[15] E. Trélat. Contrôle optimal. Mathématiques Concrètes. [Concrete Mathematics]. Vuibert, Paris, 2005. Théorie \& applications. [Theory and applications].

[16] R. Triggiani. Controllability and observability in banach space with bounded operators. SIAM Journal on Control, 13(2):462-491, 1975.

[17] G. Turinici and H. Rabitz. Optimally controlling the internal dynamics of a randomly oriented ensemble of molecules. Phys. Rev. A, 70:063412, Dec 2004.

[18] V. A. Ugrinovskii. Robust controllability of linear stochastic uncertain systems. Automatica J. IFAC, 41(5):807-813, 2005.

[19] E. Zuazua. Averaged control. Automatica, 50(12):3077 - 3087, 2014. 